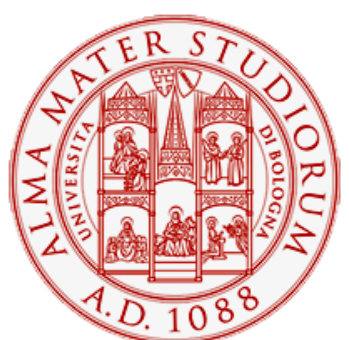

Alma Mater Studiorum - Università di Bologna DEPARTMENT OF ECONOMICS

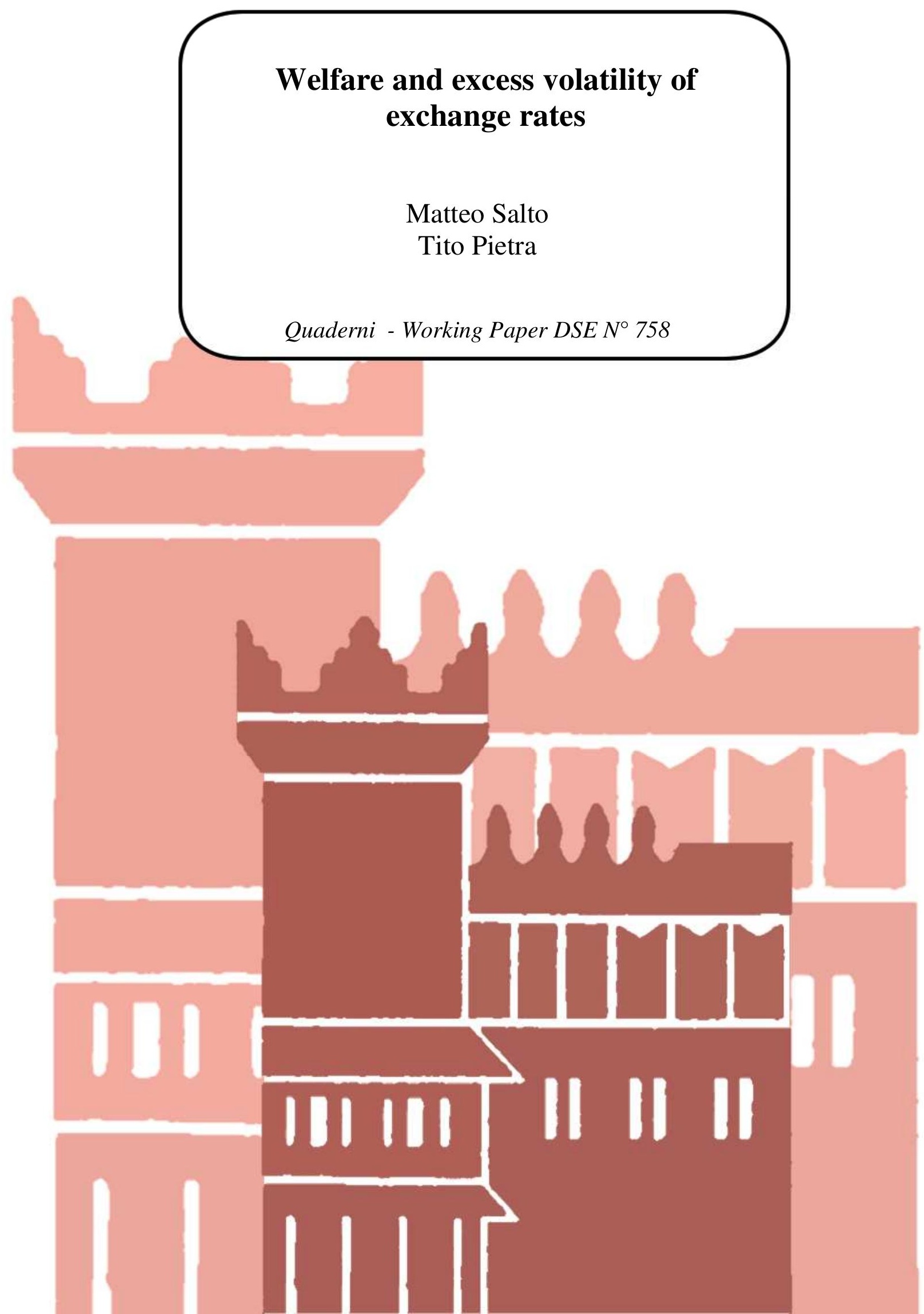




\title{
Welfare and excess volatility of exchange rates
}

\author{
M. Salto ${ }^{1}$ \\ European Commission \\ E-mail: Matteo.SALTO@ec.europa.eu \\ and \\ T. Pietra \\ Università di Bologna - D.S.E. \\ E-mail: tito.pietra@unibo.it
}

Version: June 10, 2011

\begin{abstract}
We study the properties of a GEI model with nominal assets, outside money (injected into the economy as in Magill and Quinzii [17]), and multiple currencies. We analyze the existence of monetary equilibria and the structure of the equilibrium set under two different assumptions on the determination of the exchange rates. If currencies are perfect substitutes, equilibrium allocations are indeterminate and, generically, sunspot equilibria exist. Generically, given a nonsunspot equilibrium, there are Pareto improving (and Pareto worsening) sunspot equilibria associated with an increase in the volatility of the future exchange rates. We interpret this property as showing that, in general, there is no clearcut effect on welfare of the excess volatility of exchange rates, even when due to purely extrinsic phenomena.
\end{abstract}

Key Words: Incomplete markets, exchange rates, real indeterminacy, sunspots, Pareto efficiency

JEL Classification: D52

\section{INTRODUCTION}

The analysis of the determinants of exchange rates is still largely an open issue. Since Meese and Rogoff [21], it is well known that standard theoretical models perform very poorly in predicting the short-run behavior of exchange rates (see also Cheung, Chinn and Pascual [7]). Moreover, it is often claimed that exchange rates exhibit excess volatility, and that this may entail a welfare cost. Starting with Kareken and Wallace [15], several theoretical contributions have studied economies where exchange rates may be indeterminate and may be affected by extrinsic uncertainty. Their seminal paper considers a two-country overlapping generation environment with no uncertainty and where the two currencies are perfect substitutes. They show that equilibrium exchange rates are indeterminate. Their key result survives in cash-in-advance environments with infinitely living agents and where currencies play a pure transactional role, see King, Wallace and Weber [16]. Manuelli

\footnotetext{
${ }^{1}$ This is a completely revised and extended version of a paper by the first author, "Real indeterminacy and nominal exchange rates", part of his Ph.D. dissertation at the Universitè Catholique de Louvain. He wishes to thank for suggestions and encouragements H. Polemarchakis. Careful suggestions by a referee of this journal have been particularly helpful. The usual disclaimers apply. The second author aknowledges the financial support of MIUR - PRIN 2009. The views expressed here are those of the authors and do not involve the EU Commission.
} 
and Peck [18] consider a stochastic OLG model, and do not restrict the analysis to stationary equilibria. They show that stationary allocations can be associated to nonconstant exchange rate processes, so that it is impossible to derive general conclusions on the efficiency of equilibria looking at the volatility of exchange rates. Bear in mind that excess volatility is essentially innocuous in terms of welfare, in the two classes of economies just mentioned. An additional contribution along these lines is Russel [28]. He extends the analysis allowing for the existence of two real assets in an economy where price levels are inversely correlated (hence, returns on money holdings positively correlated) with the returns from the real assets of the same country. In this context, the author shows existence and Pareto-inferiority of quasi-sunspot equilibria. Finally, Alonso [1] considers a Shubik trading-posts model with uncertainty, and shows that equilibrium exchange rates are indeterminate and affect welfare when currencies are perfect substitutes. The relevance of perfect substitutability is confirmed by the literature on search models of money, which analyzes the conditions for the coexistence of several currencies and the endogenous choice of currencies in a multi-country environment. In particular, Martin [19] shows that, in a two-country, cash-in-advance model with fixed costs for the use of foreign currencies, the exchange rate is determinate if there are enough frictions to endogenously prevent all the agents from trading in more than one currency i.e. if fixed costs are high enough.

Our starting point is a version of Magill and Quinzii [17], simplified by assuming that outside money has a pure transactional role. ${ }^{2}$ A previous application of their approach to study open economies is in Neumeyer [22]. He considers a two-period, two-country economy where each country currency is injected by forcing each agent to sell her endowment of commodities to the central exchange of her home country. Agents can then use the currency to buy from the central exchange of each country.

We basically consider the same class of economies, extending the analysis to an arbitrary number of commodities (in Neumeyer [22] there is just one good in each country). We mainly study the opposite polar case, where each agent can choose the central exchange where to sell her endowment. This corresponds to the frictionless case of the literature. If, at least some, agents can choose the exchange they sell their endowment to, the price levels in the two countries are not determined anymore by their country-specific quantity of money equation, because the two equations collapse into just one set of restrictions. This reopens the door to the possibility of obtaining real indeterminacy of the equilibrium set. More precisely, under this formulation of the model, monetary equilibria exhibit as many degrees of real indeterminacy as the number of tomorrow states of nature. ${ }^{3} \mathrm{We}$ restrict the analysis to two-period, two-country economies. Obviously, this has no bearing on our results. They hold as long as the number of states of nature is finite and asset markets are incomplete.

The logic behind the different properties of the equilibria associated with the two specifications of the monetary equations is transparent. Assuming perfectly free trade, absence of arbitrage opportunities in commodity trading imposes that the law of one price holds, so that commodity prices must be identical, once measured in the same currency. When, as in Neumeyer [22], there are two distinct

\footnotetext{
${ }^{2}$ For the closed economy, they show that the absence of a role of money as store of value has no substantive effects on the properties of the equilibria, so that, without any loss of generality, we can ignore this function, as long as the risk-free asset is in the asset span.

${ }^{3}$ Polemarchakis [27] studies real indeterminacy in GEI economies with exchange rates, but without outside money.
} 
monetary equations for each state of nature, the money supply in each country determines the (domestic) absolute price level. Then, the PPP condition fixes the exchange rates. This leaves no "free" endogenous parameters and, generically, leads to local uniqueness of equilibria. To the contrary, if the total money supply (converted into a common currency) determines just the world price level (in the same currency), the monetary conditions are satisfied by different combinations of price levels and exchange rates. Once we fix the period 0 numeraire and exchange rate, the remaining degrees of nominal indeterminacy generically translate into the same number of degrees of real indeterminacy. As usual in this class of economies, the two models have, in a precise sense, the same set of equilibrium allocations. With indeterminacy they are parameterized by exchange rates, which can be arbitrarily fixed. In the determined case, by the vectors of money supply. Therefore, apart from the interest of the indeterminate case per se, it is also convenient to study it because the results have a natural re-interpretation in terms of the determinate case, which is more tricky due to purely technical problems, since the rank of the matrix describing assets' payoffs is not invariant over the set of possible exchange rate vectors.

An additional motivation for the interest in the indeterminate case is that, as common in the GEI literature, real indeterminacy of equilibria allows for the generic existence of sunspot equilibria. The possible relevance of extrinsic variables in the determination of exchange rates has been previously discussed in the literature; among others, in some of the papers mentioned above. The persistence of excess volatility in exchange rates is well-established in the empirical literature. The prevailing view is that it is unambiguously bad, welfare-wise. ${ }^{4}$ When equilibria are indeterminate and sunspot equilibria exist, the appropriate notion of excess volatility is not completely obvious. ${ }^{5}$ Here, we simply mean any (mean-preserving) increase in the variance of the exchange rates across equilibria. Technically, we compare a generic nonsunspot equilibrium with sunspot equilibria characterized by a (mean-preserving) increase in the variance of the exchange rates. Section 4 shows that, given an equilibrium where sunspots do not matter, generically there are Pareto improving sunspot equilibria characterized by excess volatility of the exchange rates. Not surprisingly, there are also Pareto worsening sunspot equilibria with the same characteristics in terms of volatility. Hence, in general, it is impossible to provide any theoretically sound statement on the welfare impact of excess volatility. The same basic argument also applies, modulo some technicalities, to mean-preserving spreads of the exchange rates across pure extrinsic events.

The paper is structured as follows: we present the model in Section 2. Section 3 analyzes existence and real indeterminacy of monetary equilibria in the economy with and without extrinsic uncertainty. In particular, we establish that equilibria typically exhibit as many degrees of real indeterminacy as states of nature in the second period, independently of their nature. Section 4 is devoted to the analysis of the welfare effects of mean-preserving increases in the variance of exchange rates. We focus the analysis on sunspot equilibria, because, technically, this is the most demanding case and because sunspot volatility is a particularly strong definition of

\footnotetext{
${ }^{4}$ Explicit analyses of the welfare cost of exchange rates volatility usually conclude that they are fairly small, see, for instance, Bacchetta, and Van Wincoop [2], Devereux, and Engel [10], and Bergin, Shin, Templeton, and Tchakarov [9]. However, their definition is not in terms of purely extrinsic excess volatility.

${ }^{5}$ For instance, Citanna and Schmedders [9] define excess volatility of equilibrium asset prices as variance over and above the one associated with the complete market equilibrium.
} 
excess volatility. The same results hold in the economy without sunspots, where we can exploit full rank perturbations of the parameters of the economy. The main difference concerns the restriction on the maximum number of agents for which the results hold. The final section briefly considers the case of economies with two distinct monetary equations, i.e., the direct generalization of Neumeyer [22].

Several definitions and statements are formulated with direct reference to the sunspot economy. Their counterparts for the economy with no extrinsic uncertainty are always obvious.

\section{THE ECONOMY}

The basic structure is standard. There are two time periods with $S$ intrinsic states of nature tomorrow, indexed by $s=1, \ldots S, s=0$ denotes the first period. At each $s$, there are $L$ identical physical commodities, indexed by $\ell=1, \ldots, L$. There are $H$ agents, partitioned into two sets $H_{c}, \# H_{c} \geq 1$, where $c \in\{A, B\}$ denotes one of the two countries.

With each intrinsic state $s$, we associate $K$ extrinsic events, indexed by $k=$ $1, \ldots, K$. Thus, a state of nature is $k s \equiv \sigma, k s=11, \ldots, K S \equiv \Sigma$, and $L^{*}=(\Sigma+1) L$ is the total number of commodities. For notational convenience, and without any loss of generality, the extrinsic variables realize before the intrinsic one and are equiprobable.

With respect to consumers, we assume:

A1. $\quad$ For each $h, u_{h}: \mathbb{R}_{++}^{(S+1) L} \rightarrow \mathbb{R}$ is a $C^{2}$, Von Neumann-Morgenstern utility function with $\nabla_{x_{h}} u_{h}>>0$, and $v^{T} D_{x_{h}}^{2} u_{h} v<0$ for each $v \neq 0$. For each $\bar{x}_{h}>>0$, $c l\left\{x_{h} \in \mathbb{R}_{++}^{L(S+1)} \mid u_{h}\left(x_{h}\right) \geq u_{h}\left(\bar{x}_{h}\right)\right\} \subset \mathbb{R}_{++}^{L(S+1)}$. Moreover, $\omega_{h} \in \mathbb{R}_{++}^{L(S+1)}$. In the sunspot economy, $\omega_{h} \in \mathbb{R}_{++}^{L^{*}}$ and $\omega_{h}^{k s}=\omega_{h}^{k^{\prime} s}$, for each $s$ and each pair $k, k^{\prime}$.

The vector $e \equiv\left(e^{0}, \ldots, e^{\Sigma}\right) \in \mathbb{R}_{++}^{\Sigma+1}$ describes the exchange rates, i.e., the numbers of units of the currency of country $B$ required to buy one unit of currency $A$ at the different spots.

The sunspot-invariant vector of asset $i^{\prime}$ s payoffs in terms of home currency is $r^{i}=\left[r^{i 1}, \ldots, r^{i S}\right]$. Given $e$, the matrix of asset payoffs in terms of currency $B$ is

$$
R(e)=\left[\begin{array}{cccccc}
e^{1} r^{11} & \cdots & e^{1} r^{I_{A} 1} & r^{\left(I_{A}+1\right) 1} & \cdots & r^{I 1} \\
\vdots & \ddots & \vdots & \vdots & \ddots & \vdots \\
e^{\Sigma} r^{1 S} & \cdots & e^{\Sigma} r^{I_{A} S} & r^{\left(I_{A}+1\right) S} & \cdots & r^{I S}
\end{array}\right]
$$

$r^{k s}(e) \equiv\left[e^{k s} r^{1 s}, \ldots, r^{I s}\right] \equiv\left[e^{k s} r_{A}^{s}, r_{B}^{s}\right]$ is the vector of asset payoffs in spot $\sigma=k s$. The matrices $R_{A}(e)$ and $R_{B}$ denote the payoffs (in terms of currency $B$ ) of the two subsets of assets. In the sequel, with some abuse of notation, we will occasionally use $R(e)$ to refer to the payoff matrix in the economy without sunspots. No confusion should arise. We will always maintain:

A2. $\quad$ i. $I<S$;

ii. $\operatorname{rank} R_{A}(1)=I_{A}$ and $\operatorname{rank} R_{B}=I_{B}$, with $I \equiv I_{A}+I_{B}$, and the two submatrices of $R_{A}(1)$ and $R_{B}$ associated with event $k=1$ are in general position.

The general position assumption guarantees that, generically in $e, R(e)$ has full rank $I$ (see Lemma 1 below). For each asset $i$, the price (in national currency) is 
$q^{i}$. Absence of arbitrage opportunities requires that there is $\mu \in \mathbb{R}_{++}^{\Sigma}$ such that

$$
\left[e^{0} q^{1}, \ldots, e^{0} q^{I_{A}}, q^{I_{A}+1}, \ldots, q^{I}\right]=[\mu]^{T} R(e) .
$$

Let

$$
Y(e) \equiv\left[\begin{array}{c}
-e^{0} q^{1}, \ldots,-e^{0} q^{I_{A}}, \ldots,-q^{I} \\
R(e)
\end{array}\right] \equiv\left[\left[\begin{array}{c}
-e^{0} q_{A} \\
R_{A}(e)
\end{array}\right]\left[\begin{array}{c}
-q_{B} \\
R_{B}
\end{array}\right]\right]
$$

Assuming that there are no frictions in commodity trade, the law of one price holds. Therefore, if $p_{c}^{\sigma}$ is state $\sigma$ vector of commodity prices in country $c$, denoted in terms of its currency, it must be $e^{\sigma} p_{A}^{\sigma}=p_{B}^{\sigma}$, for each $\sigma$. Hence, we can drop from the notation $p_{B}^{\sigma}$ and set $p^{\sigma}=p_{A}^{\sigma}$. Let $\Psi(p, e)$ be the $(\Sigma+1) \times L^{*}$-dimensional matrix of commodity prices in terms of country $B$ currency,

$$
\Psi(p, e)=\left[\begin{array}{ccc}
e^{0} p^{0} & \cdots & 0 \\
\vdots & \ddots & \vdots \\
0 & \cdots & e^{\Sigma} p^{\Sigma}
\end{array}\right]
$$

Finally, $\left(M_{A}, M_{B}\right) \in \mathbb{R}_{++}^{2(S+1)}$ is the pair of (exogenous) vectors of money supply in the two countries. They are sunspot-invariant.

In the sequel, we will often treat asymmetrically some variables. We will use the superscript " $\backslash 0$ " to indicate that we are ignoring the spot 0 variables. Thus, for instance, $\Psi^{\backslash 0}(p, e)$ is given by the last $\Sigma$ rows of $\Psi(p, e)$. Similarly, $p^{\backslash L}$ denotes the price system, once we eliminate $p^{\sigma L}$, for each $\sigma$.

\subsection{Exchange rate regimes}

The monetary equations implicitly defining the exchange rate regime are obtained by an extension to a two-country model of the set-up proposed by Magill and Quinzii [17]. In a one-country economy, agents sell their initial endowments to a central exchange to acquire outside money issued by the exchange itself. In turn, they use the money so obtained to buy commodities. Moving to a two-country set-up, we have two polar cases.

In the first (the one considered in Neumeyer [22]), agents must sell their initial endowment to their home country central exchange and can buy from either one of them. This implies $\sum_{h=1}^{H_{c}} p_{c}^{k s} \omega_{h}^{s}=M_{c}^{s}$, for each $c$. Absence of commodity arbitrage imposes the additional restriction $e^{\sigma} p_{A}^{\sigma}=p_{B}^{\sigma}$. While there are some technical problems for the proof of the existence of monetary equilibria, it is fairly intuitive that equilibria, and, hence, exchange rates, are determinate. There are several alternative precise specifications of the mechanism of trading with the two central exchanges leading to the same main result: as long as there are two separate monetary eqs., equilibria are determinate.

We describe this class of economies with the monetary equations

$$
G_{d}\left(M_{A}, M_{B}, p, e\right) \equiv\left[\begin{array}{c}
e^{k s} p^{k s} \sum_{h}^{H} \omega_{h}^{s}-e^{k s} M_{A}^{s}-M_{B}^{s}, \quad \text { for each } k \text { and } s \\
p^{k s} \Sigma_{h}^{H_{A}} \omega_{h}^{s}-M_{A}^{s}, \text { for each } k \text { and } s
\end{array}\right]=0 .
$$

The opposite polar case is the one of indeterminacy. Essentially as in Kareken and Wallace [15], real indeterminacy holds when the two currencies are perfect substitutes, i.e., in our set-up, when agents can freely buy and sell from each central exchange. Given prices and exchange rates, for each agent the choice of the quantity 
of his endowment to be sold to each central exchange is indeterminate, at each equilibrium. Therefore, the only actual restrictions are the law of one price for the commodities and $e^{k s} p^{k s} \sum_{h=1}^{H} \omega_{A}^{s}=e^{k s} M_{A}^{s}+M_{B}^{s}$. We will show that, given any vector $e$, we can always find a price normalization such that a monetary equilibrium exists and that, generically, nominal indeterminacy translates into $\Sigma$ degrees of real indeterminacy. For this case, it is convenient to write the monetary eqs. as

$$
G_{n d}\left(M_{A}, M_{B}, p, e, E\right) \equiv\left[\begin{array}{c}
e^{k s} p^{k s} \Sigma_{h}^{H} \omega_{h}^{s}-e^{k s} M_{A}^{s}-M_{B}^{s}, \quad \text { for each } k \text { and } s \\
e^{k s}-E^{k s}, \text { for each } k \text { and } s
\end{array}\right]=0,
$$

for some arbitrary, exogenously given $E \in \mathbb{R}_{++}^{\Sigma+1}$.

Clearly, $G_{n d}: \mathbb{R}_{++}^{L^{*}+2(S+1)+2(\Sigma+1)} \rightarrow \mathbb{R}_{++}^{2(\Sigma+1)}$, while $G_{d}: \mathbb{R}_{++}^{L^{*}+2(S+1)+(\Sigma+1)} \rightarrow$ $\mathbb{R}_{++}^{2(\Sigma+1)}$.

It is worthwhile to stress that, generically, real indeterminacy requires perfect substitutability among currencies. For instance, equilibria of economies with different transaction costs for resident and non-resident trading with a central exchange are typically determined (see also, in a different set-up, Martin [19]). Still, the case of perfect substitutability is interesting for the reasons discussed in the introduction.

\subsection{Consumer's behavior}

Given $\pi \equiv(p, q, e)$, each agent chooses her optimal consumption bundle and portfolio $\left(x_{h}, y_{h}\right)$ solving optimization problem

$$
\text { choose }\left(x_{h}, y_{h}\right) \in \arg \max \sum_{\sigma>0} \frac{u_{h}\left(x_{h}^{0}, x_{h}^{\sigma}\right)}{K} \text { subject to } \Psi(\pi) z_{h}=Y(\pi) y_{h},
$$

where $z_{h} \equiv\left(x_{h}-\omega_{h}\right)$, i.e., under $A 1$ (and omitting the irrelevant term $\frac{1}{K}$ ), she chooses $\theta_{h} \equiv\left(x_{h}, y_{h}, \lambda_{h}\right)$ such that

$$
F O C_{h}\left(\theta_{h}, \pi\right) \equiv\left[\begin{array}{c}
D_{x_{h}}\left[\sum_{\sigma>0} u_{h}\left(x_{h}^{0}, x_{h}^{\sigma}\right)\right]-\Psi(\pi)^{T}\left[\lambda_{h}\right] \\
Y(\pi)^{T}\left[\lambda_{h}\right] \\
-\Psi(\pi) z_{h}+Y(\pi) y_{h}
\end{array}\right]=0,
$$

where $\lambda_{h} \in \mathbb{R}_{++}^{\Sigma+1}$ is the vector of Lagrange multipliers.

\subsection{Equilibrium}

An equilibrium is defined by the usual conditions, market clearing and individual optimization, and by one of the two sets of monetary equations.

Definition 1. Given $\left(M_{A}, M_{B}\right)$, a monetary equilibrium (ME) is a vector $\bar{\pi} \equiv$ $(\bar{p}, \bar{q}, \bar{e})$ with associated $(\bar{x}, \bar{y})$ such that

a. for each $h,\left(\bar{x}_{h}, \bar{y}_{h}\right)$ is an optimal solution to (1), given $\bar{\pi}$,

b. $\quad \sum_{h} \bar{z}_{h}=0$,

c. $\quad \sum_{h} \bar{y}_{h}=0$

d. $\quad G_{n d}\left(M_{A}, M_{B}, \bar{p}, \bar{e}, \bar{E}\right)=0$, for some $\bar{E} \in \mathbb{R}_{++}^{\Sigma+1}\left(\right.$ respectively, $G_{d}\left(M_{A}, M_{B}, \bar{p}, \bar{e}\right)=$ $0)$.

Given a ME allocation $\bar{x}$, sunspots do not matter if and only if, for each $h$ and $s, \bar{x}_{h}^{k s}=\bar{x}_{h}^{k^{\prime} s}$, for each $k$ and $k$.

In the sequel, we will always clarify the equilibrium we are considering by making reference to the relevant set of monetary equations. 
Remark 1. Equilibria where sunspots do non matter are induced in the natural way by the equilibria of the economy with no sunspots at all. Indeed, if $\bar{\pi} \in$ $\mathbb{R}_{++}^{L(S+1)} \times \mathbb{R}^{I} \times \mathbb{R}_{++}^{S+1}$ is a ME in the economy without sunspots, $\widehat{\pi} \in \mathbb{R}_{++}^{L(\Sigma+1)} \times$ $\mathbb{R}^{I} \times \mathbb{R}_{++}^{\Sigma+1}$, with $\left(\widehat{p}^{0}, \widehat{q}\right) \equiv\left(\bar{p}^{0}, \bar{q}\right)$ and $\left(\widehat{p}^{k s}, \widehat{e}^{k s}\right) \equiv\left(\bar{p}^{s}, \bar{e}^{s}\right)$, for each $k$ and $s$, is a ME of the sunspot economy where, evidently, sunspots do not matter.

Remark 2. If $\bar{\pi}$ is an equilibrium with associated $(\bar{x}, \bar{y})$, then, for each $\widehat{e}^{0}>0$, there is a ME $\widehat{\pi}$ with $\widehat{e}=\left(\widehat{e}^{0}, \bar{e}^{1}, . ., \bar{e}^{\Sigma}\right)$ with associated the same allocation $(\bar{x}, \bar{y})$. Simply set $\widehat{p}^{\sigma} \equiv \bar{p}^{\sigma}$ for each $\sigma \neq 0, \widehat{p}^{0} \equiv \eta \frac{\bar{e}^{0}}{\bar{c}^{0}} \bar{p}^{0}, \widehat{q}_{B} \equiv \eta \bar{q}_{B}$, and $\widehat{q}_{A} \equiv \eta \frac{\bar{e}^{0}}{\bar{e}^{0}} \bar{q}_{A}$, where $\eta>0$ is such that $\widehat{e}^{0} M_{A}^{0}+M_{B}^{0}=\eta e^{0} \bar{p}^{0} \sum_{h} \omega_{h}^{0}$. Hence, at least one degree of indeterminacy is purely nominal. In the sequel, we will mostly set $e^{0}=p^{0 L}=1$, identify $e$ with $\left(e^{11}, \ldots, e^{\Sigma}\right), p$ with $\left(p^{0 \backslash L}, \ldots, p^{\Sigma}\right)$, and drop the spot 0 monetary eqs., because they are always satisfied, modulo a renormalization.

\subsection{The space of the economies}

Most of our results hold for a generic subset of economies and, at the different stages of the analysis, we will make reference just to the set of parameters that we will need to perturb. We start defining the space of the economies in its most general form as

$$
\xi \equiv\left(\left(\ldots,\left(u_{h}, \omega_{h}\right), \ldots\right), r,\left(M_{A}^{\backslash 0}, M_{B}^{\backslash 0}\right)\right) \in \prod_{h}\left(U \times \mathbb{R}_{++}^{L(S+1)}\right) \times \Re \times \mathbb{R}_{++}^{2 S} \equiv \Xi,
$$

where $U$ is the space of functions satisfying $A 1$ above, $\Re$ is the set of (sunspotinvariant) asset payoffs such that $A 2$ holds. Given that we are ignoring the period 0 monetary equations, $\left(M_{A}^{\backslash 0}, M_{B}^{\backslash 0}\right) \in \mathbb{R}_{++}^{2 S}$.

Euclidean spaces are endowed with the standard topology, $U$ with the $C^{2}$ compact-open topology and $\Xi$ with the product topology.

To parameterize utility functions, we adopt a locally quadratic perturbation of $u_{h}$. Consider any sunspot-invariant vector $\bar{\pi}$ and let $\bar{\theta}_{h}$ be the (sunspot-invariant) agent $h^{\prime}$ s optimal choice. Pick any open ball $V_{\varepsilon}\left(\bar{x}_{h}\right) \subset \mathbb{R}_{++}^{L(S+1)}$ such that $V_{2 \varepsilon}\left(\bar{x}_{h}\right) \subset$ $\mathbb{R}_{++}^{L(S+1)}$, and any smooth bump function $\psi\left(x_{h}\right)$, such that $\psi\left(x_{h}\right)=1$ if $x_{h} \in$ $V_{\varepsilon}\left(\bar{x}_{h}\right), \psi\left(x_{h}\right)=0$ if $x_{h} \notin c l V_{2 \varepsilon}\left(\bar{x}_{h}\right)$. Replace $u_{h}\left(x_{h}\right)$ with

$$
\widetilde{u}_{h}\left(x_{h}\right)=u_{h}\left(x_{h}\right)+\psi\left(x_{h}\right)\left(a x_{h}-\frac{1}{2}\left[x_{h}\right]^{T} D\left[x_{h}\right]\right),
$$

where $a \in \mathbb{R}^{L(S+1)}$, while $D$ is a $(L(S+1) \times L(S+1))$ symmetric matrix. Evidently, given $\psi\left(x_{h}\right)$, for $\|a\|$ and $\|D\|$ small enough, $\widetilde{u}_{h}\left(x_{h}\right)$ satisfies $A 1$.

In the sequel, a subset of $\Xi$ is generic if it is open and dense. When utility functions are fixed, we strengthen the notion, requiring that the subset has full Lebesgue measure, too.

We consider both the economy without sunspots and the one with sunspots. In the analysis, all the fundamental parameters are obviously sunspot-invariant.

\section{ECONOMIES WITH $G_{N D}()=$.}

\subsection{Equilibria in the economy without extrinsic uncertainty}

If we impose $G_{n d}()=$.0 , a ME always exists. It suffices to fix $e=\bar{E} \in \mathbb{R}_{++}^{S+1}$, and to impose a normalization $\bar{e}^{s} M_{A}^{s}+M_{B}^{s}=\bar{e}^{s} p^{s} \sum_{h=1}^{H} \omega_{h}^{s}$, for each $s>0$, and 
$\left(p^{0}, q\right) \in \Delta$. Then, we can exploit the fixed point argument of Werner [31], for instance. Modulo a renormalization of $\left(p^{0}, q\right)$ (so that the monetary equations hold at $s=0$, too), this allows us to show the existence of a ME (see Thm. 1). Moreover, under A2, and for a generic (see Lemma 1 below) choice of the vector $\bar{E}$, rank $R(\bar{E})=I$. For economies in this set, generic regularity of equilibria follows by a standard argument. Hence, we simply summarize these properties.

First, it is fairly intuitive that, under A2, generically $\operatorname{rank} R(E)=I$. For completeness, we formally establish this property in the next Lemma. The proof is in Appendix.

Lemma 1. Under A2, there is an open, dense subset of $\mathbb{R}_{++}^{S}$, of full Lebesgue measure, $\mathcal{E}$, such that, for each $E \in \mathcal{E}, \operatorname{rank} R(E)=I$, in the economy without sunspots.

Using Lemma 1, the next Thm. summarizes existence and regularity properties of ME. Its proof follows by a standard argument, that we omit.

THEOREM 1. Under the maintained assumptions, in the economy associated with $G_{n d}()=$.0 , there is a $M E$. At each $\bar{E} \in \mathcal{E}$, and for each $\xi \in \Xi^{\prime}(\bar{E})$, an open, dense subset of $\Xi, M E$ are locally described by a finite collection of smooth functions $\left\{\left(\pi^{j}(\xi, E), \theta_{1}^{j}(\xi, E), \ldots, \theta_{H}^{j}(\xi, E)\right\}\right.$, for $j=1, \ldots, J$.

To analyze real indeterminacy of equilibria, we use the definition proposed in Geanakoplos and Mas-Colell [14]: The set of equilibrium allocations has $S$ degrees of real indeterminacy if it contains the image of a nonempty, open subset of $\mathbb{R}^{S}$ under a smooth, injective map. The basic logic of our argument is the same as in $[14$, Thm. 2]: different vectors $(\bar{e}, \widehat{e})$ typically induce different spans of the payoff matrix in terms of real purchasing power. Generically, this translates into indeterminacy of the equilibrium allocation. Here, changes of the vector $e$ act on the span of the matrix $R(e)$ through two channels: their direct effect on $\left\langle R(e)>^{6}\right.$ and their effect on commodity prices, due to the monetary eqs. Hence, their proof needs to be adjusted to keep into account this last feature of our model. The details are in Appendix.

THEOREM 2. Under the assumptions of Thm. 1, in the economy associated with $G_{n d}()=$.0 , if $H>I$ and $S>2 I$, there is an open, dense set of economies $\Xi(\bar{E}) \subset \Xi^{\prime}(\bar{E})$ such that the set of equilibrium allocations has $S$ degrees of real indeterminacy.

Remark 3. Most of the results on GEI with variable asset prices show that there are $(S-1)$ degrees of real indeterminacy. Here, the "free" parameter $E$ affects the span of the return matrix acting differently on the payoffs of the assets of the two countries. This explains why we have one more degree of real indeterminacy than usual. A similar result holds for economies with mixed (partly real, partly nominal) payoffs (see Geanakoplos and Mas-Colell [14, Thm. 2] and Pietra [23]). Also, we require that the number of degrees of incompleteness is sufficiently large $(S>2 I)$. This is a standard assumption for economies with both real and nominal assets (see, again, Thm. 2 in [14] and [23]). It is quite likely that this last restriction could be relaxed at some cost in terms of technicalities.

\footnotetext{
${ }^{6}$ Our set-up is closer to the one of their Thm. 2, where they consider economies with both real and nominal assets.
} 
Remark 4. Regularity of ME only requires endowment perturbations, so that it holds for a generic subset of endowments of full Lebesgue measure. To the contrary, our proof of real indeterminacy also exploits utility perturbations, so that the generic set $\Xi(\bar{E})$ is an open and dense subset of $\Xi$.

Remark 5. The vector $E$ plays a double role. In Thm. 1, we fix it and determine the set of regular economies $\Xi^{\prime}(\bar{E})$, which depends upon $\bar{E}$. In Thm. 2, given $\xi \in \Xi(\bar{E})$, we consider the set of equilibria (locally) associated with the economies described (among the other parameters) by $E$ itself.

Remark 6. Dealing with a two-country economy, it is common to assume that there is a risk-free (in terms of home currency) asset in each country. This is a typical assumption made in most open macroeconomic models. With the monetary equations $G_{n d}()=0,. M E$ exist for each asset structure such that $A 2$ holds. In Lemma 1, we just require that $R_{A}(1)$ and $R_{B}$ are in general position (not that $\left[R_{A}(1) R_{B}\right]$ is). Hence, the result holds if we require the existence of a risk-free asset in each country. The proofs of Thm. 1 and 2 do not require perturbations of the payoffs of assets 1 and $I_{A}+1$ either, so that they hold if we impose this additional restriction. This also implies that there is no loss of generality in our ignoring the role of outside money as a store of value.

\subsection{Sunspot equilibria}

Here, we consider the existence of sunspot equilibria. This can also be seen as preliminary work for the following section. We study economies in the generic (as we will show in Lemma 2) set $\Xi(\bar{E})$ for which nonsunspot $M E$ are regular in the sunspot extension of the economy, and study the real effects of changes in the vector $E$. Our argument is purely local. Consider an economy $\xi \in \Xi(\bar{E})$ with a regular equilibrium (in the economy without sunspots) such that $R(\bar{E})$ has full rank and $M E$ display $S$ degrees of real indeterminacy. This $M E$ naturally induces a nonsunspot equilibrium. We show that, modulo a sunspot-invariant perturbation of $\left(u_{1}, \omega_{1}\right)$, this nonsunspot equilibrium is regular in the sunspot economy and that sunspot ME display $\Sigma$ degrees of real indeterminacy, so that sunspots matter.

Compared with previous results in sunspot economies with nominal assets, (see, Cass [5], Pietra [24, 25], and Suda, Tallon and Villanacci [30]), here we have an additional difficulty, related to the monetary eqs. The most convenient approach is to drop the last $\Sigma$ eqs. of $G_{n d}^{\backslash 0}(\pi ; \xi, \bar{E})$, eliminating the variable $\bar{E}$. We will still use $\bar{E}$ just to describe the sets of economies which depend upon the selection of a specific value of the exchange rates. From now on we call $G_{n d}^{\backslash 0}(\pi ; \xi)$ the set of global monetary equations $e^{\sigma} p^{\sigma} \Sigma_{h}^{H} \omega_{h}^{s}-e^{\sigma} M_{A}^{s}-M_{B}^{s}=0$. Fix $\xi$ and define the system of equations

$$
\Theta(\pi ; \xi) \equiv\left[\begin{array}{c}
\zeta^{\backslash L}(\pi ; \xi) \\
G_{n d}^{\backslash 0}(\pi ; \xi)
\end{array}\right]=0,
$$

where $\zeta^{\backslash L}($.$) is the system of market clearing equations for each commodity and$ asset, but commodity $\sigma L$, for each $\sigma$. Evidently, $\zeta^{\backslash L}(\pi ; \xi)=0$ implies market clearing for all the commodities and all the assets. $\Theta(\pi ; \xi)$ is given by $\left(L^{*}+I-1\right)$ equations in $\left(L^{*}+I+\Sigma-1\right)$ endogenous variables. Given $\xi$, a ME is a solution to $\Theta(\pi ; \xi)=0$ and is regular if and only if $D_{(p, q)} \Theta(\theta, \pi ; \xi)$ has full rank, where

$$
D_{(p, q)} \Theta(\pi ; \xi)=\left[\begin{array}{cc}
D_{(p, q)} \zeta^{\backslash L}(\pi ; \xi) \\
{\left[\Psi^{\backslash 0}\left(\sum_{h} \omega_{h}, e\right)\right.} & 0
\end{array}\right]
$$


and where $\Psi^{\backslash 0}\left(\sum_{h} \omega_{h}, e\right)$ is the $\left(\Sigma \times L^{*}\right)$ matrix with generic non-zero coefficients $e^{k s} \sum_{h} \omega_{h}^{s}$. The standard approach must be adjusted to take care of this additional set of $\Sigma$ equations. Here, and in the sequel, we start with an arbitrary economy $(\bar{u}, \bar{\omega})$ and one of its nonsunspot equilibria associated with some $\bar{e} \in \mathcal{E}$, $\bar{\pi}=(\bar{p}, \bar{q}, \bar{e})$. We perturb utility functions and endowments, constructing an economy $\widetilde{\xi} \equiv(\widetilde{\omega}, \widetilde{u})$ such that $\bar{\pi}$ is still an equilibrium of $\widetilde{\xi}$ and with a very simple structure of the matrix $D_{(p, q)} \Theta(\pi ; \widetilde{\xi})$ at $\bar{\pi}$, so that we can easily establish that its rank is full. Given $\widetilde{u}$ (which can be taken arbitrarily close to $\bar{u}$ ), we then construct $\widehat{\omega}$ arbitrarily close to $\bar{\omega}$ and such that $\bar{\pi}$ is an equilibrium for each economy $\left(\widetilde{u}, \omega^{\mu}\right), \omega^{\mu}=\mu \widehat{\omega}+(1-\mu) \widetilde{\omega}$ for some $\mu \in[0,1]$. The structure of the matrix $D_{(p, q)} \Theta\left(\pi ; \widetilde{u}, \omega^{\mu}\right)$ shows that, at $\bar{\pi}, \operatorname{det} D_{(p, q)} \Theta\left(\pi ; \widetilde{u}, \omega^{\mu}\right)$ is a nontrivial polynomial in $\mu$. Hence, at $\bar{\pi}, \operatorname{det} D_{(p, q)} \Theta\left(\pi ; \widetilde{u}, \omega^{\mu}\right)=0$ has a finite number of solutions, and $\operatorname{det} D_{(p, q)} \Theta\left(p, q ; \widetilde{u}, \omega^{\mu}\right) \neq 0$ for $\mu$ in some generic subset of $[0,1]$. This immediately implies that the subset of economies with a regular nonsunspot equilibrium is dense. Its openness follows immediately. The details are in Appendix. Here, we use perturbations of the utility functions, $\Xi \equiv \prod_{h}\left(U \times \mathbb{R}_{++}^{L(S+1)}\right)$ and a subset of $\Xi$ is generic if it is open and dense.

Lemma 2. Assume $A 1$ and $A 2$, fix $\bar{E} \in \mathcal{E}$, and consider the economy with $G_{n d}^{\backslash 0}()=$.0 . Then, there is an open, dense subset $\Xi(\bar{E}) \subset \Xi$ such that, for each $(\omega, u) \in \bar{\Xi}(\bar{E})$, all the nonsunspot equilibria associated with $\bar{E}$ are regular in the sunspot economy.

Given this result, existence and real indeterminacy of sunspot $M E$ follow immediately.

Theorem 3. Assume $H>I, S>2 I, A 1$ and A2, and consider the economy with $G_{n d}^{\backslash 0}()=$.0 . Then, there is an open, dense set of economies $\widehat{\Xi}(\bar{E}) \subset \Xi$ such that the set of equilibrium allocations has $\Sigma$ degrees of real indeterminacy in the sunspot economy. Moreover, for each regular nonsunspot equilibrium associated with $\bar{E}$, there is an open nbd $V(\bar{E})$ such that, for each $\widehat{E} \in V(\bar{E})$ with $\widehat{E}^{k s} \neq \widehat{E}^{k^{\prime}}$, for some $k, k^{\prime}, s, x^{k s}(\widehat{E}) \neq x^{k^{\prime}}(\widehat{E})$, i.e., sunspots matter.

\section{EXCESS VOLATILITY OF EXCHANGE RATES AND WELFARE}

In this section we study the welfare effects of extrinsic uncertainty. As mentioned in the introduction, excess volatility of exchange rates and its welfare implications have been discussed in the literature from both the empirical and the theoretical viewpoint. In our set-up, the notion of excess volatility can be formalized in at least three different ways. One could argue that, per se, real effects of extrinsic uncertainty mean excess volatility of the endogenous variables, because, by very definition, it is uncertainty unrelated to the fundamentals. However, given a nonsunspot equilibrium, there are sunspots equilibria with a lower value of the variance of the exchange rates. Therefore, to identify sunspot equilibria with "excess volatility" presents some ambiguity. To avoid it, we consider a nonsunspot equilibrium and define excess volatility as a mean-preserving increase of the variance of the exchange rates. A somewhat stronger notion could be defined in terms of mean-preserving spreads of the exchange rates prevailing in the nonsunspot economy. This would require us to consider increases in the variability of the exchange 
rates across extrinsic events, for each intrinsic event, keeping the expected value of the exchange rate invariant for each $s$. Looking at the proof, it will become clear that, modulo some differences in the details, our result still holds in this case. The only substantive difference is that the maximum number of agents such that Thm. 4 holds decreases from $H<[(L-1) S-2]$ to $H<(L-3) S$, because the two eqs. $\operatorname{var}(e)=\overline{\operatorname{var}}$ and $\operatorname{Exp}(e)=\overline{\operatorname{Exp}}$ are replaced by $2 S$ eqs., a pair of similar restrictions for each intrinsic event $s>0$ (see Corollary 1).

Therefore, the question we address in this section is: Pick a nonsunspot equilibrium with vector of exchange rates $\bar{e}$. Is there any general welfare implication of an increase in the variance of $e, \operatorname{var}(e)$, which preserves its expected value, $E(\bar{e})$ ?

Define the map

$\Phi(\pi ; \xi, \bar{V}, \overline{v a r}, \overline{\operatorname{Exp}}) \equiv\left[\begin{array}{c}\Theta(\pi ; \xi) \\ \sum_{\sigma} \frac{u_{1}\left(x_{1}^{\sigma}(.)\right)}{K}-\bar{V}_{1} \\ \vdots \\ \sum_{\sigma} \frac{u_{H}\left(x_{H}^{\sigma}(.)\right)}{K}-\bar{V}_{H} \\ \operatorname{var}(e)-\overline{v a r} \\ \operatorname{Exp}(e)-\overline{\operatorname{Exp}}\end{array}\right] \equiv\left[\begin{array}{c}\Theta(\pi ; \xi) \\ W(\pi ; \xi, \bar{V}, \overline{v a r}, \overline{\operatorname{Exp}})\end{array}\right]=0$.

Given a nonsunspot ME, for an appropriate choice of the vector $\bar{V}$ and of $(\overline{v a r}, \overline{\operatorname{Exp}})$, we obtain a solution to $\Phi()=$.0 . The previous question can then be reformulated as: In general, is there a solution to $\Phi(\pi ; \xi, \widehat{V}, \widehat{v a r}, \overline{\operatorname{Exp}})=0$ for some vector $(\widehat{V}, \widehat{v a r})>>\left(\bar{V}_{h}, \overline{v a r}\right)$ ? As well known (see, Cass and Citanna [6], Citanna, Kajii, and Villanacci [8], and Elul $[12,13])$, a sufficient condition for a positive answer is that $D_{\pi} \Phi($.$) has full rank (in our set-up, at the nonsunspot equilibrium). When$ this condition is satisfied, given $\xi$, by the implicit function theorem, we can locally find a solution to $\Phi(\pi ; \xi, \widehat{V}, \widehat{v a r}, \widehat{\operatorname{Exp}})=0$, for each vector $(\widehat{V}, \widehat{\operatorname{var}}, \widehat{\operatorname{Exp}})$ in some open neighborhood of $(\bar{V}, \overline{v a r}, \overline{E x p})$. In particular, we can find it for each $\widehat{V}>>\bar{V}, \widehat{v a r}>\overline{v a r}$, and $\widehat{\operatorname{Exp}}=\overline{\operatorname{Exp}}$, so that a mean-preserving increase in the variance of future exchange rates leads to a Pareto improvement. Obviously, we also have Pareto worsening mean-preserving increases in their variance (simply, choose $\widehat{V}<<\bar{V})$.

We focus on the comparison of sunspot vs. nonsunspot equilibria. Abstracting from sunspots, one could wonder if variations in the structure of exchange rates satisfying, for instance, some additional restrictions on their covariances with some other relevant variables, could have well-defined welfare implications. Our conjecture is that the answer is, in general, negative.

Our approach can be directly (and more simply) applied to economies without sunspots. To introduce additional restrictions on the allowed changes in exchange rates would just alter some of the details of the argument.

In this section we do not need to perturb $\left(M_{A}, M_{B}\right)$ and $r$ (satisfying $A 2$ ). Hence, we set $\Xi=\prod_{h}\left(U \times \mathbb{R}_{++}^{L(S+1)}\right)$.

In Appendix, we formally establish the next Thm.

Theorem 4. Let $2 \leq H \leq[(L-1) S-2]$. Under the maintained assumptions, 
there is an open, dense subset $\widetilde{\Xi}(\bar{E}) \subset \Xi$, such that, for each $(u, \omega) \in \widetilde{\Xi}(\bar{E})$, there is a nonsunspot equilibrium such that there are both Pareto improving and Pareto worsening mean-preserving increases in the variance of the exchange rates.

Its result can be strengthened, considering mean preserving spreads of exchange rates in the sunspot economy. This just requires a minor change in the proof.

Corollary 1. Let $2 \leq H \leq(L-3)$ S. Under the maintained assumptions, there is an open, dense subset $\widetilde{\Xi}(\bar{E}) \subset \Xi$, such that, for each $(u, \omega) \in \widetilde{\Xi}(\bar{E})$, given a nonsunspot equilibrium, there are both Pareto improving and Pareto worsening mean-preserving spreads of the exchange rates in the sunspot economy.

Remark 7. In the proof of Thm. 4, we fix a particular vector $\bar{e}=\bar{E}$ and the result holds generically for one of the nonsunspot $\mathrm{ME}$ associated with $\bar{E}$. One could also strengthen the result, showing that it actually holds generically at each nonsunspot ME associated with $\bar{E}$. Moreover, if we are willing to restrict the analysis to bounded and bounded away from zero subsets of the space $\mathcal{E}$, the same result holds generically at each nonsunspot ME. Both arguments are straightforward, but tedious, and therefore omitted.

Remark 8. A similar result holds when one considers economies with no extrinsic uncertainty as long as $2 \leq H \leq S-2$, i.e., as long as the number of "free variables", the $S$ exchange rates, exceeds the number of additional equations $(H+2)$.

Remark 9. Given a nonsunspot equilibrium $\bar{e}$, the characteristics of the vector $\widehat{e}$ with $\operatorname{var}(\widehat{e})>\operatorname{var}(\bar{e}), \operatorname{Exp}(\widehat{e})=\operatorname{Exp}(\bar{e})$ and associated with a, say, Pareto superior allocation $\widehat{x}$ are entirely dictated by our choice of $\widehat{v a r}$ and $\left(\widehat{V}_{1}, \ldots, \widehat{V}_{H}\right)$. Generally speaking, at the Pareto superior allocation sunspots matter, because, in the sunspot economy, the argument works for a number of agents larger than in the nonsunspot economy, given that we have a larger number of "free parameters", $\Sigma$ instead of $S$.

The details of the proof of the Thm. are a little cumbersome, but the basic idea is quite simple and develops the approach put forth in Pietra [26]. Let

$$
D_{\pi} \Phi(\pi ; \xi, \bar{V}, \overline{v a r}, \overline{E x p}) \equiv\left[\begin{array}{ccc}
D_{p} \zeta^{\circ}(\pi ; \xi) & D_{q} \zeta^{\circ}(\pi ; \xi) & D_{e} \zeta^{\circ}(\pi ; \xi) \\
\Psi^{\backslash 0}\left(\sum_{h} \omega_{h}, e\right) & 0 & -\operatorname{diag}\left[\frac{M_{B}^{s}}{e^{k s}}\right] \\
D_{p} W(\pi ; \xi) & D_{q} W(\pi ; \xi) & D_{e} W(\pi ; \xi)
\end{array}\right]
$$

where $\zeta^{\circ}($.$) is the system of excess demand functions for all the assets and for a$ collection of $\left(L^{*}-\Sigma-1\right)$ commodities, selected so that $\zeta^{\circ}()=$.0 implies market clearing for all the assets and commodities. ${ }^{7}$

By direct computation, and using the sequence of budget constraints, it is easy

\footnotetext{
${ }^{7}$ In the proof of Thm. 4, we drop different physical commodities in different states of nature.
} 
to check that

$$
D_{\pi} W(.)=\left[\begin{array}{ccccc}
\cdots & -\lambda_{1}^{\sigma}\left[e^{\sigma} z_{1}^{\sigma}\right] & \ldots & -\lambda_{1}^{0}\left[y_{1}\right] & {\left[\ldots,-\frac{\lambda_{1}^{\sigma}}{e^{\sigma}}\left[0, r_{B}^{s}\right] y_{1}, \ldots\right]} \\
\vdots & \vdots & \vdots & \vdots & \vdots \\
\cdots & -\lambda_{1}^{\sigma}\left[e^{\sigma} z_{H}^{\sigma}\right] & \cdots & -\lambda_{H}^{0}\left[y_{H}\right] & {\left[\ldots,-\frac{\lambda_{1}^{\sigma}}{e^{\sigma}}\left[0, r_{B}^{s}\right] y_{H}, \ldots\right]} \\
0 & \ldots & \ldots & \ldots & {\left[\ldots, \frac{2\left(e^{\sigma}-\operatorname{Exp}(e)\right)}{\Sigma}, \ldots\right]} \\
0 & \ldots & \ldots & \ldots & {\left[\ldots, \frac{1}{\Sigma}, \ldots\right]}
\end{array}\right] .
$$

As in the previous section, we replace the actual economy, $\bar{\xi}$, with an economy $(\bar{u}, \widehat{\omega})$ having the same equilibrium prices, $\bar{\pi}$, and allocation, but with an associated matrix $D_{\pi} W($.$) having a very simple structure. We then show that, for (\bar{u}, \widehat{\omega})$, $D_{\pi} \Phi($.$) has full rank. This may require a sunspot-invariant perturbation of some$ utility functions and endowments. An argument similar to the one used to establish Lemma 2, shows that this implies that, given $\bar{\xi}$ and any open neighborhood $V(\bar{\xi})$, we can always find $\xi^{\prime} \in V(\bar{\xi})$ such that $\operatorname{det} D_{\pi} \Phi\left(. ; \xi^{\prime}\right) \neq 0$. This is the key result. The details are in Appendix.

Remark 10. Thm. 4 can also be exploited to show that, in economies with real assets (and, possibly, a unique equilibrium), there still exist sunspot equilibria which are Pareto superior to the nonsunspot equilibrium. The Thm. rests on the real indeterminacy of ME because this property is crucial to guarantee that, generically, sunspot equilibria exist. However, real indeterminacy of ME and welfare consequences of excess volatility are two logically distinct issues. This is also because sunspot equilibria may exist in GEI characterized by local determinacy of equilibria, where, as we have seen, exchange rates are a purely nominal variable. In economies where ME are locally unique, sunspot equilibria characterized by excess volatility of exchange rates can still exist and the same results on their welfare consequences can still hold. Pick an economy with two nominal assets. Given a nonsunspot equilibrium associated with some (sunspot invariant) $\bar{e}$, there are sunspot equilibria associated with two sunspot-dependent vectors $e^{a}, e^{b}$ such that $x\left(e^{a}\right)$ Pareto dominates $x(\bar{e})$ which, in turn, dominates $x\left(e^{b}\right)$. Following, essentially, the technique developed in Mas-Colell [20], if there are enough commodities, there is an economy with real assets such that the three equilibrium allocations $\left(x(\bar{e}), x\left(e^{a}\right), x\left(e^{b}\right)\right)$ are equilibria of its sunspot extension.

\section{ECONOMIES WITH $G_{D}()=$.}

With the condition $G_{d}()=$.0 , the vector $e$ has to be endogenously determined at the ME, and there is a critical subset of values of $e$ inducing a collapse of rank of $R(e)$. Therefore, the system of excess demand functions is discontinuous over the set of possible exchange rates. Hence, we need a different existence argument. In our framework, the pseudo-equilibrium map includes the monetary eqs. Our proof of existence is a minor modification of the one in Duffie and Shafer [11]. The only difference is in the argument used to show that the derivative of the projection map from the pseudo-equilibrium manifold to the space of the economies is nonsingular. Here we just report the existence result. ${ }^{8}$ Thm. 5 refers to economies without

\footnotetext{
${ }^{8}$ The complete proof is in Salto, Pietra [29].
} 
sunspots and utility functions are fixed, so that $\Xi \equiv \mathbb{R}_{++}^{H L(S+1)} \times \Re \times \mathbb{R}_{++}^{2 S}$.

THEOREM 5. Under the maintained assumptions, in the economy associated with $G_{d}()=$.0 , there is an open, dense subset $\Xi_{d} \subset \Xi$, of full Lebesgue measure, such that, for each $\xi \in \Xi_{d}$, there is a $M E$, with rank $R(\bar{e})=I$. Moreover, at each $\xi \in \Xi_{d}, M E$ are locally described by a finite collection of smooth functions, $\left\{\left(\pi^{j}(\xi),\left(\theta_{1}^{j}(\xi), \ldots, \theta_{H}^{j}(\xi)\right)\right)\right\}$, for $j=1, \ldots, J$.

The existence argument, given the condition $G_{d}()=$.0 , requires a perturbation of the asset structure. Hence, it may fail if we impose additional restrictions, such as the existence of a risk-free asset denoted in each currency. ${ }^{9}$

For given $\left(M_{A}, M_{B}\right)$, equilibria are locally unique for economies with $G_{d}()=$.0 . However, in a well-specified sense, the analysis of the previous sections for economies with $G_{n d}()=$.0 carries over to this class of economies, because, locally, the structure of the equilibrium set is the same, in allocation space. Indeed, real indeterminacy of $\mathrm{ME}$ for the economies with $G_{n d}()=$.0 translates into effectiveness of monetary policy for the ones with $G_{d}()=$.0 . This intuitive relationship is made precise in the following Proposition (the proof is straightforward and, hence, omitted).

Proposition 1. Given $\left(\bar{M}_{A}, \bar{M}_{B}\right)$, let $\bar{\pi}$ be a $M E$ associated with $G_{d}()=$.0 . Then, $\bar{\pi}$ is also a ME associated with $G_{n d}()=$.0 , given $\bar{E}=\bar{e}$. Moreover, if $(p(\widehat{E}), q(\widehat{E}))$ is a $M E$ associated with $G_{n d}(., \widehat{E})=0$, there is a vector $\left(M_{A}(\widehat{E}), M_{B}(\widehat{E})\right)$ such that $(p(\widehat{E}), q(\widehat{E}), \widehat{E})$ is an equilibrium associated with $G_{d}\left(M_{A}(\widehat{E}), M_{B}(\widehat{E}), p, e\right)=$ 0 .

This fact is quite convenient, because it allows us to study the properties of ME for one class of economies and to extend the results (modulo a reinterpretation) to the other. Evidently, if we are considering a sunspot equilibrium of the economy defined by $G_{n d}()=$.0 , the vectors of money supply required to support it as an equilibrium of the economy defined by $G_{d}()=$.0 will have to be sunspot-dependent. Consequently, the discussion concerning the welfare effects of excess volatility must be reinterpreted in this set-up as effects of randomness of monetary policy unrelated to the "fundamentals" $\left\{\left(\ldots,\left(u_{h}, \omega_{h}\right), \ldots\right), r,\right\}$ of the economy. Evidently, the results of section 4 holds.

Corollary 2. Let $2 \leq H \leq[(L-1) S-2]$. Under the maintained assumptions, there is an open, dense subset $\widetilde{\Xi} \subset \Xi$, such that, for each $(u, \omega) \in \widetilde{\Xi}$, there are nonsunspot equilibria such that there are both Pareto improving and Pareto worsening mean-preserving increases in the variance of the exchange rates induced by sunspot-dependent monetary policy vectors $\left(M_{A}, M_{B}\right)$.

\section{CONCLUSIVE REMARKS}

Several properties of equilibrium exchange rates can be discussed exploiting fairly natural extensions of the GEI model with outside money and liquidity constraints proposed in Magill and Quinzii [17]. Depending upon the precise specification of the monetary equations, equilibria can be characterized by local uniqueness

\footnotetext{
${ }^{9}$ Existence of ME can be obtained modulo some arbitrarily small, appropriate adjustment of the money supply vectors. Hence, for an open and dense set of economies, not necessarily of full Lebesgue measure.
} 
or by local indeterminacy. Under the conditions sufficient to establish real indeterminacy of ME, there are also sunspot equilibria. A subset of them can be characterized as induced by excess volatility of the exchange rates. Excess volatility can induce both Pareto improvements and Pareto worsening of the equilibrium allocation.

In the Magill and Quinzii [17] class of economies, outside money is injected into the economy because agents must sell their initial endowments to a central exchange and, afterward, buy back their consumption bundle. Real indeterminacy holds if agents (actually, at least some subset of agents) may freely buy and sell from both exchanges. While, in our framework, this is a polar case, it is interesting for several reasons. One of them is that the results obtained (generically) in the indeterminate case may still hold for some sets of economies characterized by local determinacy induced by the specification of the monetary equations (i.e., by the precise mechanism of trade between agents and central exchanges).

\section{APPENDIX}

\subsection{Proofs of Lemma 1 and Theorem 2}

Proof of Lemma 1. The square matrix $\underline{R}(e)$ is constructed picking any collection of $I$ rows of $R(e)$ with at most one row for each intrinsic event. Consider the map

$$
A(d, e) \equiv \underline{R}(e)^{T} d=0,
$$

with $e \in \mathbf{B}_{M} \subset \mathbb{R}^{I}$, a ball of radius $M$, and $d \in \mathbf{S}^{I-1}$, the unit sphere in $\mathbb{R}^{I}$. Given $r, A: \mathbf{S}^{I-1} \times \mathbf{B}_{M} \rightarrow \mathbb{R}^{I}$. If $A \pitchfork 0$ at each $(d, e) \in A^{-1}(0)$, by the transversality theorem there is an open, dense subset $\overline{\mathbf{B}}_{M} \subset \mathbf{B}_{M}$, of full Lebesgue measure, such that for each $e \in \overline{\mathbf{B}}_{M}, A_{e} \pitchfork 0$ at each $d \in A_{e}^{-1}(0)$. Given that $A_{e}: \mathbf{S}^{I-1} \rightarrow \mathbb{R}^{I}$, this implies that $A_{e}^{-1}(0)=\emptyset$. We now show that $A \pitchfork 0$ at each $(e, d) \in A^{-1}(0)$. First, for each $d \neq 0$ such that $\underline{R}(e)^{T} d=0$, there are at most $\min \left\{\left(I_{A}-1\right),\left(I_{B}-1\right)\right\}$ zero coefficients $d^{s}$, because the submatrices $R_{A}(e)$ and $R_{B}$ are in general position. Hence, there are at least $\left(I_{A}+1\right)$ nonzero coefficients $d^{s}$. Evidently, $D_{(d, e)} A(d, e)$ contains the $(I \times 2(I-1))$-dimensional submatrix

$$
\begin{aligned}
& D_{(d \backslash, e \backslash)} A(d, e) \\
= & {\left[\begin{array}{c}
\left.\left[\begin{array}{ccc}
e^{1} r_{A}^{1 T} & \cdots & e^{I-1} r_{A}^{(I-1) T} \\
{\left[\begin{array}{lll}
r_{B}^{1 T} & \cdots & r_{B}^{(I-1) T}
\end{array}\right]}
\end{array}\right]\left[\begin{array}{ccc}
r_{A}^{1 T} d^{1} & \cdots & r_{A}^{(I-1) T} d^{I-1}
\end{array}\right]\right] \\
{[\cdots, 0, \cdots]}
\end{array}\right], }
\end{aligned}
$$

where we omit the columns referred to $d^{I}=1-\sum_{s=1}^{I-1} d^{i 2}$ and $e^{I}$. By the general position assumption, $\operatorname{rank}\left[\begin{array}{lll}r_{B}^{1 T} & \cdots & r_{B}^{(I-1) T}\end{array}\right]=I_{B}$. The general position assumption for $R_{A}(1)$ and the considerations above imply that the top right submatrix has full rank $I_{A}$. Hence, $\operatorname{rank} D_{(d \backslash, e)} A(d, e)=I$.

Now consider an increasing sequence, $\left\{M^{v}\right\}_{v=1}^{v=\infty}$ and the associated sequence $\mathbf{B}_{M^{v}}$, where the balls are centered on 0 . The measure of $\mathbb{R}_{++}^{S} \backslash \mathcal{E}$ is the measure of the union of a countable collection of zero measure sets. Hence, it is a null set. Denseness of $\mathcal{E}$ follows by applying the argument above to any nonempty, open subsets of $\mathbb{R}_{++}^{S} \backslash \mathcal{E}$. Openness of $\mathcal{E}$ is obvious because $\operatorname{det} \underline{R}(e)$ is a continuous function of $e$. 
Proof of Thm. 2. Pick $\bar{E}$ such that rank $R(\bar{E})=I$ and any $\xi \in \Xi^{\prime}(\bar{E})$, the set of regular economies associated with $\bar{E}$. Pick a regular equilibrium $\bar{\pi}$ associated with $\bar{e}=\bar{E}$ and $(\bar{x}, \bar{y}, \bar{\lambda})$. A standard (hence omitted) argument shows that, given $\bar{e}$, there is a generic set of economies such that

$$
\operatorname{rank}\left[R(\bar{e}) \bar{y}_{1}, \ldots, R(\bar{e}) \bar{y}_{H}\right]=I
$$

holds. The vector $\left(M_{A}, M_{B}\right)$ is fixed in the proof, and omitted as an argument of the various functions. We just assume that, for some $s, s^{\prime}$,

$$
\frac{M_{B}^{s} r^{1 s}}{M_{A}^{s} r^{\left(I_{A}+1\right) s}} \neq \frac{M_{B}^{s^{\prime}} r^{1 s^{\prime}}}{M_{A}^{s^{\prime}} r^{\left(I_{A}+1\right) s^{\prime}}}
$$

In the sequel, given $\bar{e}=\bar{E}$, we restrict the analysis to the generic subset of economies $\Xi^{\prime \prime}(\bar{E}) \subset \Xi^{\prime}(\bar{E})$ such that $(A-B)$ hold. Continuity of the equilibrium map implies that they also hold for each $e$ in some open nbd $V_{\eta}(\bar{e})$.

Fix $\left(r^{1}, r^{I_{A}+1}\right)$ and let $r \backslash=\left[r^{2}, \ldots, r^{I_{A}}, r^{I_{A}+2}, \ldots r^{I}\right]$. In the sequel, we denote $d\left[\alpha^{s}\right]$ the diagonal matrix with generic non-zero coefficient $\alpha^{s}$.

We start with an observation on the spanning of $R(e)$ in terms of real purchasing power. Given a regular equilibrium $(\bar{\pi}, \bar{x}, \bar{y})$ associated with $\bar{e}$, assume that there is $\widehat{e} \neq \bar{e}$ with associate equilibrium $(\widehat{\pi}, \bar{x}, \widehat{y})$. Then, for each $s$, the vectors $\bar{p}^{s}$ and $\widehat{p}^{s}$ must be collinear. Moreover, taking into consideration the monetary eqs., we must have

$$
\left[\frac{\widehat{e}^{s} M_{A}^{s}+M_{B}^{s}}{\bar{e}^{s} M_{A}^{s}+M_{B}^{s}}\right] \bar{e}^{s} \bar{p}^{s \ell}=\widehat{e}^{s} \widehat{p}^{s \ell}, \text { for each } s \ell .
$$

Hence, under $(A)$, invariance of the equilibrium allocation at $\bar{e}$ and $\widehat{e}$ requires

$$
<R(\bar{e})>=<d\left[\frac{\bar{e}^{s} M_{A}^{s}+M_{B}^{s}}{\widehat{e}^{s} M_{A}^{s}+M_{B}^{s}}\right] R(\widehat{e})>
$$

so that the span of the payoff matrix, in terms of real purchasing power, is the same at the two equilibria. Thus, it must be that each column $i$ of $R(\bar{e})$ can be expressed as a linear combination of the columns of $d\left[\frac{\bar{e}^{s} M_{A}^{s}+M_{B}^{s}}{\widehat{e}^{s} M_{A}^{s}+M_{B}^{s}}\right] R(\widehat{e})$, with weights $\left(\beta_{i}^{1}, \ldots, \beta_{i}^{I}\right)$. Without any loss of generality, consider the two columns $\left(1, I_{A}+1\right)$. For some $\left(\beta_{1}, \beta_{I_{A}+1}\right) \in \mathbb{R}^{2 I}$, it must be

$$
A\left(\beta, \widehat{e} ; \bar{e}, r^{\backslash}\right) \equiv\left[\begin{array}{c}
{\left[\bar{e} r^{1}\right]-d\left[\begin{array}{c}
\bar{e}^{s} M_{A}^{s}+M_{B}^{s} \\
\widehat{e}^{s} M_{A}^{s}+M_{B}^{s}
\end{array}\right] R(\widehat{e}) \beta_{1}} \\
{\left[r^{I_{A}+1}\right]-d\left[\frac{\bar{e}^{s} M_{A}^{s}+M_{B}^{s}}{\widehat{e}^{s} M_{A}^{s}+M_{B}^{s}}\right] R(\widehat{e}) \beta_{I_{A}+1}}
\end{array}\right]=0 .
$$

$A_{(\bar{e}, r \backslash)}(\beta, \widehat{e})$ maps $\mathbb{R}^{S+2 I}$ into $\mathbb{R}^{2 S}$. Given that $S>2 I$, by a standard application of the transversality theorem, if $A(\beta, \widehat{e} ; \bar{e}, r \backslash) \pitchfork 0$ at each solution $A(\beta, \widehat{e} ; \bar{e}, r \backslash)=$ 0 such that $\widehat{e} \neq \bar{e}$, then, for a generic choice of $\left(\bar{e}, r^{\backslash}\right)$, there is no solution to $A_{(\bar{e}, r \backslash)}(\beta, \widehat{e})=0$ with $\widehat{e} \neq \bar{e}$. By direct computation, at a solution to $A(\beta, \widehat{e} ; \bar{e}, r \backslash)=$ 
0

$$
\begin{aligned}
D_{\bar{e}} A(.)= & {\left[\begin{array}{c}
d\left[r^{1 s}\right]-d\left[\frac{M_{A}^{s}}{\widehat{e}^{s} M_{A}^{s}+M_{B}^{s}}\right] R(\widehat{e}) \beta_{1} \\
-d\left[\frac{M_{A}^{s}}{\widehat{e}^{s} M_{A}^{s}+M_{B}^{s}}\right] R(\widehat{e}) \beta_{I_{A}+1}
\end{array}\right] } \\
= & {\left[\begin{array}{c}
\left.d\left[r^{1 s}\right]-d\left[\frac{M_{A}^{s}}{\bar{e}^{s} M_{A}^{s}+M_{B}^{s}}\right] d\left[\frac{\bar{e}^{s} M_{A}^{s}+M_{B}^{s}}{\widehat{e}^{s} M_{A}^{s}+M_{B}^{s}}\right] R(\widehat{e}) \beta_{1}\right] \\
-d\left[\frac{M_{A}^{s}}{\bar{e}^{s} M_{A}^{s}+M_{B}^{s}}\right] d\left[\frac{\bar{e}^{s} M_{A}^{s}+M_{B}^{s}}{\widehat{e}^{s} M_{A}^{s}+M_{B}^{s}}\right] R(\widehat{e}) \beta_{I_{A}+1}
\end{array}\right] } \\
= & {\left[\begin{array}{c}
d\left[r^{1 s}\right]-d\left[\frac{\bar{e}^{s} M_{A}^{s} r^{1 s}}{\bar{e}^{s} M_{A}^{s}+M_{B}^{s}}\right] \\
-d\left[\frac{\left.M_{A}^{s} r^{(I}+1\right) s}{\bar{e}^{s} M_{A}^{s}+M_{B}^{s}}\right]
\end{array}\right]=\left[\begin{array}{c}
d\left[\frac{M_{B}^{s} r^{1 s}}{\bar{e}^{s} M_{A}^{s}+M_{B}^{s}}\right] \\
-d\left[\frac{\left.M_{A}^{s} r^{(I}+1\right) s}{\bar{e}^{s} M_{A}^{s}+M_{B}^{s}}\right]
\end{array}\right] . }
\end{aligned}
$$

Hence,

$$
\begin{aligned}
& \operatorname{rank} D_{(\bar{e}, r \backslash)} A(.)
\end{aligned}
$$

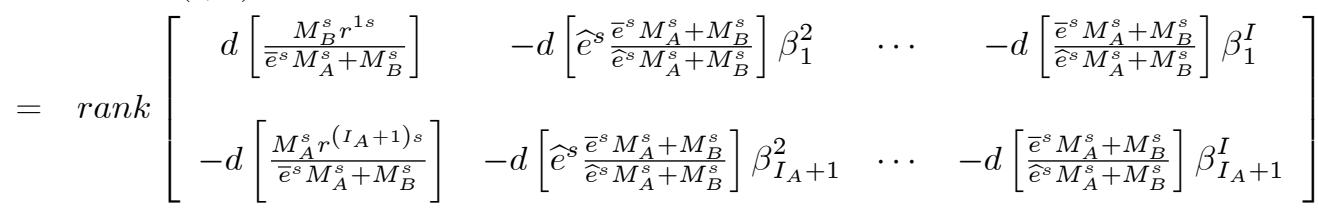

$$
\begin{aligned}
& =\operatorname{rank}\left[\begin{array}{cccc}
d\left[\frac{M_{B}^{s} r^{1 s}}{M_{A}^{s} r^{\left(I_{A}+1\right) s}}\right] & -I_{S} \beta_{1}^{2} & \cdots & -I_{S} \beta_{1}^{I} \\
-I_{S} & -I_{S} \beta_{I_{A}+1}^{2} & \cdots & -I_{S} \beta_{I_{A}+1}^{I}
\end{array}\right] \text {, }
\end{aligned}
$$

where $I_{S}$ is the $S \times S$ identity matrix. Define the last matrix as $\bar{A}($.$) . If \operatorname{rank} \bar{A}($.) $<$ $2 S$, it must be $\left[\beta_{1}\right]=\alpha\left[\beta_{I_{A}+1}\right]$ for some $\alpha \neq 0$, and $\frac{M_{B}^{s} r^{1 s}}{M_{A}^{s} r^{\left(I_{A}+1\right) s}}=-\alpha$ for each $s$. This is impossible, because, by construction, $(B)$ above holds, so that, for some $s, s^{\prime}, \frac{M_{B}^{s} r^{1 s}}{M_{A}^{s} r^{\left(I_{A}+1\right) s}} \neq \frac{M_{B}^{s^{\prime}} r^{1 s^{\prime}}}{M_{A}^{s^{\prime}} r^{\left(I_{A}+1\right) s^{\prime}}}$. Hence, $\operatorname{rank} \bar{A}()=.2 S$ and, given $\left(r^{1}, r^{I_{A}+1}\right)$, there is an open, dense set $(e, r \backslash)$ of full Lebesgue measure such that $A_{(\bar{e}, r \backslash)}(\beta, \widehat{e}) \pitchfork 0$ at each solution $A_{(\bar{e}, r \backslash)}(\beta, \widehat{e})=0$ with $\widehat{e} \neq \bar{e}$. Thus, $A_{(\bar{e}, r \backslash)}(\beta, \widehat{e})=0$ has no solution $\widehat{e} \neq \bar{e}$, for each economy $\xi$ in some generic set that we can identify with $\Xi$ " $(\bar{E})$ itself. A standard argument completes the proof.

\subsection{Proofs of Lemma 2 and Theorem. 3}

Here, and in the sequel, we will use extensively the structure of the matrix $D_{\pi} \zeta($.$) and it is convenient to report its most relevant properties (see Balasko and$ Cass [3]).

Fact 1. Under the maintained assumptions, for each $h$,

$$
D_{\pi} z_{h}(\pi ; \xi)=-\left[B_{h}^{1} B_{h}^{2}\right] D_{\pi} F O C_{h}(.),
$$

where $B_{h}^{1}$ is a negative semi-definite matrix. Moreover, the submatrix obtained from $B_{h}^{1}$ by deleting the $(\Sigma+1)$ rows and columns indexed by $\sigma \ell(\sigma)$, for each collection 
$(\ell(0), \ldots, \ell(\Sigma))$ is negative-definite. Hence, it has full rank $\left(L^{*}+I-\Sigma-1\right)$. Also, $\left[\begin{array}{ll}B_{h}^{1} & B_{h}^{2}\end{array}\right]$ does not depend directly upon $z_{h}$ and $y_{h}$.

Throughout the proof, $p^{0 L}=e^{0}=1$. As above, $d\left[\alpha^{k s}\right]$ denotes a diagonal matrix, with typical nonzero coefficient $\alpha^{k s}$.

Consider the sunspot economy. By direct computation,

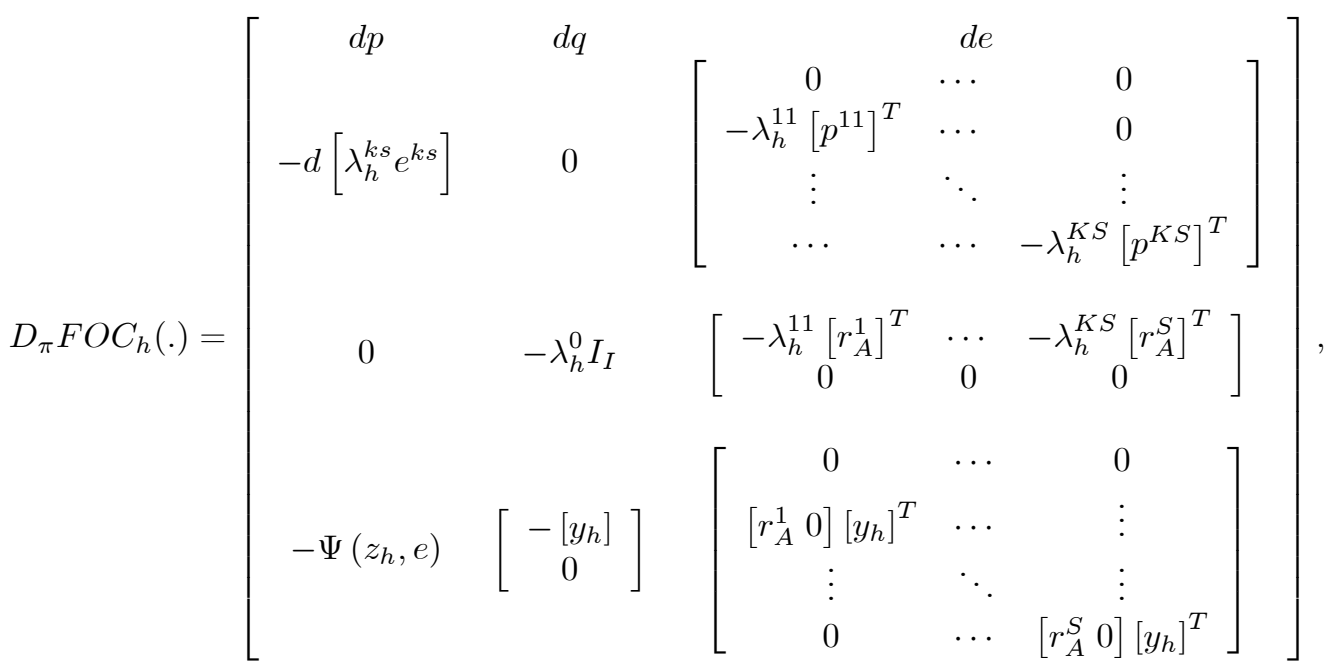

where the first row reports the variables we are differentiating with respect to. $\Psi\left(z_{h}, e\right)$ has the same structure as $\Psi(p, e)$, but for $z_{h}^{k s \ell}$ replacing $p^{k s \ell}$.

Proof of Lemma 2. Consider an economy $(\bar{u}, \bar{\omega})$ and any $\bar{e}$ such that $\operatorname{rank} R(\bar{e})=$ $I$. Let $\bar{\pi}$ be any associated nonsunspot equilibrium. We need to show that, generically, $D_{(p, q)} \Theta($.$) has full rank at \bar{\pi}$, in the sunspot economy. Bear in mind that all the derivatives are computed at $\bar{\pi}$. By direct computation, and rearranging columns, we can rewrite it as

$$
D_{(p, q)} \Theta(.) \equiv\left[\begin{array}{ccc}
D_{(p \backslash L, q)} \zeta^{\backslash L}(.) & D_{\left(p^{1 L}, \ldots, p^{\Sigma L}\right)} \zeta^{\backslash L}(.) \\
{\left[\Psi^{\backslash 0}\left(\sum_{h} \omega_{h}^{\backslash L}, e\right)\right.} & 0] & d\left[e^{k s} \sum_{h} \omega_{h}^{s L}\right]
\end{array}\right] .
$$

Given $(\bar{u}, \bar{\omega})$, consider a sunspot-invariant perturbation of $\omega_{1}$, with $\widetilde{\omega}_{1}^{s L} \equiv\left(\bar{\omega}_{1}^{s L}-\frac{\widetilde{\delta}}{\bar{e}^{s}}\right)$ and $\widetilde{\omega}_{1}^{s \ell} \equiv \bar{\omega}_{1}^{s \ell}$, for each $s \ell \neq s L$ and $s=0$. For each $h>1, \widetilde{\omega}_{h} \equiv \bar{\omega}_{h} . \widetilde{x}$ is also identical to $\bar{x}$, but for $\widetilde{x}_{1}^{s L} \equiv\left(\bar{x}_{1}^{s L}-\frac{\widetilde{\delta}}{\bar{e}^{s}}\right)$, for each $s$. Define the following (locally) quadratic perturbation of $u_{1}\left(x_{1}\right)$ :

$$
\widetilde{u}_{1}\left(x_{1}\right) \equiv \bar{u}_{1}\left(x_{1}\right)+\psi\left(x_{1}\right)\left(a x_{1}-\frac{1}{2}\left[x_{1}\right]^{T} D x_{1}\right),
$$

where $D \equiv\left[\left.D_{x}^{2} \bar{u}_{1}\left(x_{1}\right)\right|_{\widetilde{x}_{1}}-\left.D_{x}^{2} \bar{u}_{1}\left(x_{1}\right)\right|_{\bar{x}_{1}}\right]$ is a symmetric, square, $L(S+1)$-dimensional matrix, while $a \equiv\left[\left.\nabla_{x_{1}} \bar{u}_{1}\left(x_{1}\right)\right|_{\bar{x}_{1}}-\left.\nabla_{x_{1}} \bar{u}_{1}\left(x_{1}\right)\right|_{\widetilde{x}_{1}}+D \widetilde{x}_{1}\right]$ is an $L(S+1)$-dimensional vector. It follows that

1. $\left.\nabla_{x_{1}} \widetilde{u}_{1}\left(x_{1}\right)\right|_{\widetilde{x}_{1}}=\left.\nabla_{x_{1}} \bar{u}_{1}\left(x_{1}\right)\right|_{\bar{x}_{1}}$,

2. $\left.D_{x}^{2} \widetilde{u}_{1}\left(x_{1}\right)\right|_{\widetilde{x}_{1}}=\left.D_{x}^{2} \bar{u}_{1}\left(x_{1}\right)\right|_{\bar{x}_{1}}$. 
Hence, $\left.D_{\theta_{1}}^{2} F O C_{1}\left(. ; \widetilde{u}_{1}, \widetilde{\omega}_{1}\right)\right|_{\left(\widetilde{x}_{1}, \bar{\pi}\right)}=\left.D_{\theta_{1}}^{2} F O C_{1}\left(. ; \bar{u}_{1}, \bar{\omega}_{1}\right)\right|_{\left(\bar{x}_{1}, \bar{\pi}\right)}$. Moreover, for $\widetilde{\delta}$ small enough, $(\widetilde{u}, \widetilde{\omega})$ satisfies $A 1$. It is easy to check that, for each $\widetilde{\delta}$ small enough, $\bar{\pi}$ is a nonsunspot $M E$ of the economy $(\widetilde{u}, \widetilde{\omega})$, with associated allocation $\widetilde{x}$.

Fix any open nbd $V(\bar{u}, \bar{\omega})$. For each $\widetilde{\delta}$ sufficiently small, $(\widetilde{u}, \widetilde{\omega}) \in V(\bar{u}, \bar{\omega})$. We now show that there is an economy $\left(\omega^{\mu}, \widetilde{u}\right)$ arbitrarily close to $(\widetilde{u}, \widetilde{\omega})$, hence to $(\bar{u}, \bar{\omega})$, and such that $\operatorname{det} D_{(p, q)} \Theta\left(. ; \widetilde{u}, \omega^{\mu}\right) \neq 0$.

Given $\widetilde{\delta} \neq 0$, fix $u=\widetilde{u}$ and define the economy with $\widehat{\omega}_{1}=\widetilde{x}_{1}$ and $\widehat{\omega}_{h}=\bar{x}_{h}$, for each $h>1$. Evidently, $\bar{\pi}$ and $(\widetilde{x}, 0, \bar{\lambda})$ is a nonsunspot $M E$ of $(\widetilde{u}, \widehat{\omega})$, with $\widetilde{z}_{h}=0$. Moreover, at this equilibrium, $D_{(p, q)} F O C_{h}\left(. ; \widetilde{u}_{h}, \widehat{\omega}_{h}\right)$ does not depend, directly, upon the endowments, and, therefore, upon the value of $\widetilde{\delta}$, and

$$
D_{(p \backslash L, q)} \zeta^{\backslash L}(.)=-\sum_{h} B_{h}^{1 \backslash L}\left[\begin{array}{cccc}
\ddots & \cdots & \cdots & \cdots \\
\vdots & -\lambda_{h}^{\sigma} I_{(L-1)} & \vdots & \vdots \\
\vdots & & \ddots & \vdots \\
\cdots & \cdots & \cdots & -\lambda_{h}^{0} I_{I}
\end{array}\right]
$$

is a full rank matrix, since it is the sum of negative-definite matrices. In the economy $(\widetilde{u}, \widehat{\omega})$, let $\widehat{C} \equiv-\left[\left.D_{(p \backslash L, q)} \zeta^{\backslash L}(.)\right|_{\bar{\pi}}\right]^{-1} D_{p^{L}} \zeta^{\backslash L}(. ; \widetilde{u}, \widehat{\omega})$, so that

$$
\left[D_{\left(p^{\backslash L}, q\right)} \zeta^{\backslash L}(. ; \widetilde{u}, \widehat{\omega}) \quad D_{p^{L}} \zeta^{\backslash L}(. ; \widetilde{u}, \widehat{\omega})\right]\left[\begin{array}{c}
\widehat{C} \\
I_{\Sigma}
\end{array}\right]=[0]
$$

and define the $\Sigma \times \Sigma$ matrix

$$
\widehat{F} \equiv\left[\left[\Psi^{\backslash 0}\left(\widehat{\omega}^{\backslash L}, \bar{e}\right) \quad 0\right] \widehat{C}+d\left[\bar{e}^{k s} \sum_{h}^{H} \widehat{\omega}_{h}\right]\right] .
$$

By construction of $\widehat{\omega}$,

$$
\begin{aligned}
& \widehat{F}=\left[\left[\begin{array}{ll}
\Psi^{\backslash 0}\left(\bar{\omega}^{\backslash L}, \bar{e}\right) & 0
\end{array}\right] \widehat{C}+d\left[\bar{e}^{k s} \sum_{h}^{H} \bar{\omega}_{h}\right]\right]-d\left[\bar{e}^{k s} \frac{\widetilde{\delta}}{\bar{e}^{s}}\right] \\
& \equiv G-\widetilde{\delta} I_{\Sigma},
\end{aligned}
$$

by sunspot invariance of $\bar{e}$. Given that $G$ does not depend upon $\widetilde{\delta}$, we can arbitrarily perturb $\widetilde{\delta}$ without affecting it. Evidently, if $\operatorname{det} \widehat{F}=0, \widetilde{\delta}$ is an eigenvalue of $G$. Given that eigenvalues are locally unique, $\widehat{F}$ has full $\underset{\widetilde{\delta}}{\operatorname{rank}} \Sigma$, modulo an arbitrarily small perturbation $\widetilde{\delta}$. Then, for an appropriate value of $\widetilde{\delta}$, at the associated $(\widetilde{u}, \widehat{\omega})$,

$$
\begin{aligned}
& \operatorname{rank} D_{(p, q)} \Theta(.)=\operatorname{rank} D_{(p, q)} \Theta(.)\left[\begin{array}{cc}
I_{L^{*}+I-\Sigma-1} & \widehat{C} \\
0 & I_{\Sigma}
\end{array}\right]
\end{aligned}
$$

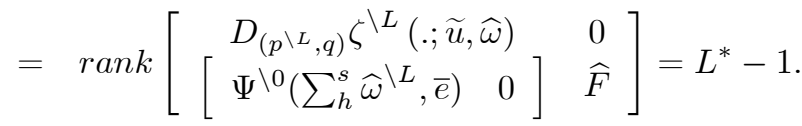

Thus, given any open nbd $V(\bar{u}, \bar{\omega})$, we can pick $\widetilde{\delta}$ and construct $(\widetilde{u}, \widetilde{\omega}) \in V(\bar{u}, \bar{\omega})$ and $(\widetilde{u}, \widehat{\omega})$ so that

$$
\operatorname{det} D_{(p, q)} \Theta(. ; \widetilde{u}, \widehat{\omega}) \neq 0 \text {. }
$$


Given $\widetilde{u}$, consider the set of endowments $\omega^{\mu} \equiv(\mu \widehat{\omega}+(1-\mu) \widetilde{\omega})$, with $\mu \in[0,1]$. First, $\bar{\pi}$ is an equilibrium for each $\mu$, with associated $\left(\widetilde{x}_{h}, y_{h}^{\mu}, \bar{\lambda}_{h}\right)$ for each $h$, where $y_{h}^{\mu}=(1-\mu) \widetilde{y}_{h}$. Secondly, the last $\Sigma$ rows of the matrix $D_{(p, q)} \Theta\left(\bar{\pi} ; \widetilde{u}, \omega^{\mu}\right)$ are $\mu$-invariant. Finally, the first $\left(L^{*}+I-\Sigma-1\right)$ rows are given by

$D_{(p \backslash L, q)} \zeta^{\backslash L}\left(\bar{\pi} ; \widetilde{u}, \omega^{\mu}\right)=-\sum_{h} B_{h}^{1 \backslash L}\left[\begin{array}{cccc}\ddots & \cdots & \cdots & \cdots \\ \vdots & -\lambda_{h}^{\sigma} I_{(L-1)} & \vdots & \vdots \\ \vdots & \cdots & \ddots & \vdots \\ \cdots & \cdots & \cdots & -\lambda_{h}^{0} I_{I}\end{array}\right]-\sum_{h} B_{h}^{2 \backslash L} M_{h}(\mu)$

where $M_{h}(\mu)$ is given by the last $(\Sigma+1)$ rows of $D_{\pi} F O C_{h}($.$) , after dropping the$ columns referred to commodity $L$ at each spot. It is clearly a linear function of $\mu$. Hence, $\operatorname{det} D_{(p, q)} \Theta\left(\bar{\pi} ; \widetilde{u}, \omega^{\mu}\right)$ is a polynomial in $\mu$ and $\operatorname{det} D_{(p, q)} \Theta\left(\bar{\pi} ; \widetilde{u}, \omega^{\mu}\right) \neq 0$ at $\mu=1$. Given that non-trivial polynomials have a finite set of zeros, we can always find $\mu$ such that $\left(\widetilde{u}, \omega^{\mu}\right)$ is arbitrarily close to $(\widetilde{u}, \widetilde{\omega})$, hence to $(\bar{u}, \bar{\omega})$, and such that $D_{(p, q)} \Theta\left(. ; \widetilde{u}, \omega^{\mu}\right)$ has full rank. This establishes the density part. Openness follows immediately because $\operatorname{det} D_{(p, q)} \Theta\left(. ; \widetilde{u}, \omega^{\mu}\right)$ is a continuous function.

By Thm. 1, given $\bar{E}$, for all the economies in some generic subset $\Xi^{\prime}(\bar{E})$, for each $(u, \omega) \in \Xi^{\prime}(\bar{E})$, all nonsunspot equilibria are regular and there is a finite number of them. By repeating the argument above for each equilibrium, and taking the intersection of the finite collection of open, dense sets so obtained, we construct a generic set $\bar{\Xi}(\bar{E})$ such that all the nonsunspot equilibria associated with $\bar{E}$ are regular in the sunspot economy.

Proof of Thm. 3. The spanning argument exploited to show the real indeterminacy result in Thm. 1 basically works in the sunspot economy, too. Pick a nonsunspot equilibrium associated with $\bar{e} \in \mathbb{R}_{++}^{\Sigma}$. Assume that the associated equilibrium is regular in the sunspot economy and that it satisfies $(A-B)$ defined in the proof of Thm. 2, and

$$
\sum_{i=1}^{I_{A}} r^{s i} \bar{y}_{h}^{i} \neq^{s} \bar{p}^{s} \bar{z}_{h}^{s}\left(\frac{\bar{e}^{s} M_{A}^{s}}{\bar{e}^{s} M_{A}^{s}+M_{B}^{s}}\right) \text {, for each } s \text { and } h .
$$

Pick any two vectors $\widehat{e} \neq \widetilde{e}, \widehat{e}, \widetilde{e} \in V_{\eta}(\bar{e})$ with associated allocations $\widehat{x}$ and $\widetilde{x}$. Given that there must be some $k(s) s$ such that $\widehat{e}^{k(s) s} \neq \widetilde{e}^{k(s) s}$, the pure spanning argument above still applies, when restricted to the vectors $\left[e^{11}, e^{1 s-1}, e^{k(s) s}, e^{1 s+1}, \ldots, e^{1 S}\right]$. Hence, it must be $\widehat{x} \neq \widetilde{x}$. The real indeterminacy result follows immediately.

Moreover, consider any $\widehat{e}$ such that, for some $s$ and $k, k^{\prime}, \widehat{e}^{k s} \neq \widehat{e}^{k^{\prime} s}$. If $\widehat{z}_{h}^{k s}=\widehat{z}_{h}^{k^{\prime}} s$, it must be that $\widehat{p}^{k s}$ and $\widehat{p}^{k^{\prime} s}$ are collinear and, taking into account the monetary eqs., that

$$
\widehat{e}^{k^{\prime} s} \widehat{p}^{k^{\prime} s} \widehat{z}_{h}^{k s}=\widehat{e}^{k s} \widehat{p}^{k s} \widehat{z}_{h}^{k s} \frac{\widehat{e}^{k^{\prime} s} M_{A}^{s}+M_{B}^{s}}{\widehat{e}^{k s} M_{A}^{s}+M_{B}^{s}} .
$$

The budget constraints in the two states require

$$
\begin{aligned}
& \sum_{i=1}^{I_{A}} \widehat{e}^{k^{\prime} s} r^{s i} \widehat{y}_{h}^{i}+\sum_{i=I_{A}+1}^{I} r^{s i} \widehat{y}_{h}^{i}= \widehat{e}^{k^{\prime} s} \widehat{p}^{k^{\prime} s} \widehat{z}_{h}^{k s}=\widehat{e}^{k s} \widehat{p}^{k s} \widehat{z}_{h}^{k s} \frac{\widehat{e}^{k^{\prime} s} M_{A}^{s}+M_{B}^{s}}{\widehat{e}^{k s} M_{A}^{s}+M_{B}^{s}} \\
& \text { and } \\
& \sum_{i=1}^{I_{A}} \widehat{e}^{k s} r^{s i} \widehat{y}_{h}^{i}+\sum_{i=I_{A}+1}^{I} r^{s i} \widehat{y}_{h}^{i}=\widehat{e}^{k s} \widehat{p}^{k s} \widehat{z}_{h}^{k s} .
\end{aligned}
$$


Hence,

$$
\begin{aligned}
\sum_{i=1}^{I_{A}} \widehat{e}^{k^{\prime} s} r^{s i} \widehat{y}_{h}^{i}-\sum_{i=1}^{I_{A}} \widehat{e}^{k s} r^{s i} \widehat{y}_{h}^{i} & =\widehat{e}^{k s} \widehat{p}^{k s} \widehat{z}_{h}^{k s}\left(\frac{\widehat{e}^{k^{\prime} s} M_{A}^{s}+M_{B}^{s}}{\widehat{e}^{k s} M_{A}^{s}+M_{B}^{s}}-1\right) \\
\sum_{i=1}^{I_{A}} r^{s i} \widehat{y}_{h}^{i} & =\widehat{p}^{k s} \widehat{z}_{h}^{k s}\left(\frac{\widehat{e}^{k s} M_{A}^{s}}{\widehat{e}^{k s} M_{A}^{s}+M_{B}^{s}}\right) .
\end{aligned}
$$

By $(C)$ above, at $\bar{e}$ this condition is violated at each $s$. Hence, it must also be violated for each $\widehat{e}$ sufficiently close to $\bar{e}$, so that, locally, sunspots matter at each $\widehat{e}$ such that, for some $s, k, k^{\prime}, \widehat{e}^{k s} \neq \widehat{e}^{k^{\prime} s}$.

If one takes as a reference point, as we are doing, a regular nonsunspot equilibrium, if $\widehat{e} \neq \widetilde{e}$ but, for each $s, \widehat{e}^{k s}=\widetilde{e}^{k^{\prime} s}$ for some permutation of the indexes $k, k^{\prime}$, we clearly obtain that $\widehat{x}=\widetilde{x}$ modulo a permutation of the allocation across extrinsic events. It is far from obvious that one should consider the two equilibrium allocations as different, from a substantive viewpoint. To avoid this issue, take as starting point a regular sunspot equilibrium with $\bar{e}^{k s} \neq \bar{e}^{k^{\prime} s}$ for each $k, k^{\prime}$ and each $s$. The same argument implies that, locally, given $\widehat{e} \neq \widetilde{e}$, the associated $\widehat{x}, \widetilde{x}$ satisfy $\widehat{x} \neq \widetilde{x}$ and it cannot be $\widehat{e}^{k s}=\widetilde{e}^{k^{\prime} s}$ for each $k, k^{\prime}$ and each $s$. This rules out the possibility that $\widehat{x}^{k s}=\widetilde{x}^{k^{\prime}} s$, for each $s$ and modulo some permutation of the indexes $k$.

\subsection{Proof of Theorem 4 and Corollary 1}

Restrict the analysis to economies with a regular sunspot equilibrium, i.e., to $\xi \in \bar{\Xi}(\bar{E})$. As motivated in the text, the proof reduces to show that, for each $\xi$ in some generic subset of $\bar{\Xi}(\bar{E})$, there is a regular sunspot equilibrium such that

$$
\operatorname{rank} D_{\pi} \Phi(.) \equiv \operatorname{rank}\left[\begin{array}{c}
D_{\pi} \zeta^{\circ}(.) \\
D_{\pi} G_{n d}^{\backslash 0}(.) \\
D_{\pi} W(.)
\end{array}\right]=\left(L^{*}+I-\Sigma-1\right)+\Sigma+(H+2) .
$$

We start with a fairly obvious result.

Fact 2. Without any loss of generality, the economy $(\bar{u}, \bar{\omega}) \equiv \bar{\xi} \in \bar{\Xi}(\bar{E})$ has a regular nonsunspot equilibrium $\bar{\pi}$, with associated $\bar{\theta}$, such that $\frac{\bar{\lambda}_{1}^{1}}{\bar{\lambda}_{1}^{2}} \neq \frac{\bar{\lambda}_{H}^{1}}{\bar{\lambda}_{H}^{2}}$.

Proof of Fact 2. $\quad$ Given $\bar{\pi}$, replace $\bar{u}_{1}\left(x_{h}\right)$ with

$$
u_{1}^{\gamma}\left(x_{h}\right)=\bar{u}_{1}\left(x_{1}\right)+\psi\left(x_{1}\right) \sum_{s \ell} \gamma^{s} \bar{\lambda}_{1}^{s} \bar{e}^{s} \bar{p}^{s \ell} x_{1}^{s \ell}
$$

for some vector $\gamma$ such that $\left[\ldots, \bar{\lambda}_{1}^{s}\left(1+\gamma^{s}\right), \ldots\right] Y(\bar{e})=0$ and $\frac{\bar{\lambda}_{1}^{1}\left(1+\gamma^{1}\right)}{\bar{\lambda}_{1}^{2}\left(1+\gamma^{2}\right)} \neq \frac{\bar{\lambda}_{H}^{1}}{\bar{\lambda}_{H}^{2}}$. Given that $\frac{\partial u_{1}^{\gamma}\left(x_{1}\right)}{\partial x_{1}^{s \ell}}=\frac{\partial \bar{u}_{1}\left(x_{h}\right)}{\partial x_{1}^{s \ell}}+\gamma^{s} \bar{\lambda}_{1}^{s} \bar{e}^{s} \bar{p}^{s \ell},\left(\bar{x}_{1}, \bar{y}_{1}\right)$, with Lagrange multipliers $\left[\bar{\lambda}_{1}^{s}\left(1+\gamma^{s}\right)\right]$, is 1 's optimal choice at $\bar{\pi}$. Given $\left(\bar{x}_{1}, \psi\left(x_{1}\right)\right), u_{1}^{\gamma}\left(x_{1}\right)$ satisfies $A 1$ above, for $\gamma$ sufficiently small. The claim follows immediately.

We now replace the actual economy $\bar{\xi}$ with a new economy $\xi(\alpha)$, with the same equilibrium $\bar{\pi}$ and such that the associated matrix $D_{\pi} W($.$) has a very simple$ structure. 
Given $\bar{\xi} \in \bar{\Xi}(\bar{E}), \xi(\alpha) \equiv(\bar{u}, \omega(\alpha))$ is defined as follows: For agent $1, \omega_{1}^{s \ell}(\alpha) \equiv$ $\bar{x}_{1}^{k s \ell}$ for all the commodities but $(11,1 L, 21,2 L), \omega_{1}^{s 1}(\alpha) \equiv \bar{x}_{1}^{s 1}-\alpha$, and $\omega_{1}^{s L}(\alpha) \equiv$ $\bar{x}_{1}^{s L}+\frac{\bar{p}^{s 1}}{\bar{p}^{s L}} \alpha$, for $s=1,2$.

Define a one-to-one map $f:\{2, \ldots, H-1\} \rightarrow\{\ldots,(s 1, \ldots, s(L-1)), \ldots\} \backslash\{11,21\}$, associating with each agent $h, 1<h<H-1$, one commodity $s \ell, \ell \neq L$ and $s \ell \neq 11$, $s \ell \neq 21$. For each agent $h, 1<h<H, \omega_{h}^{s \ell}(\alpha) \equiv \bar{x}_{h}^{k s \ell}$ for all the commodities, but commodity $f(h), \omega_{h}^{f(h)}(\alpha) \equiv \bar{x}_{h}^{f(h)}-\alpha$, and, for the same $s, \omega_{h}^{s L}(\alpha) \equiv \bar{x}_{h}^{s L}+\frac{\bar{p}^{f(h)}}{\bar{p}^{s L}} \alpha$. Hence, agent $h, 1<h<H$, buys one unit of good $\ell$ paying in terms of commodity $L$ at $s$ such that $s \ell=f(h)$. Set $\omega_{H}(\alpha) \equiv \bar{x}_{H}+\sum_{h<H}\left(\bar{x}_{h}^{s L}-\omega_{h}(\alpha)\right)$. For each $\alpha$, $\omega(\alpha)$ is sunspot-invariant and $\sum_{h} \omega_{h}(\alpha) \equiv \sum_{h} \bar{\omega}_{h}$. Evidently, $\bar{\pi}$ is a ME of the new economy $\xi(\alpha)$, associated with $\left(\bar{x}_{h}, 0, \bar{\lambda}_{h}\right)$, for each $h$.

For notational convenience, assume that $K=(L-1)$ and $H=(K S-2)$ (the maximum number of agents allowed). For the same reason, and without any loss of generality, assume that $\left(\bar{e}^{S}-E(\bar{e})\right)=0$. Consider the matrix $D_{\pi} \Phi($.$) , where all$ the derivatives are computed at $\bar{\pi}$.

Define the collection of $(L-1) \Sigma$ columns

$$
\pi^{d} \equiv\left\{\begin{array}{c}
\left(d p^{111}, d p^{212}, \ldots, d p^{K 1(L-1)}\right), \ldots, \quad\left(d p^{1(S-1) 1}, \ldots, d p^{(K-1)(S-1)(L-1)}\right), \\
\left(d p^{1 S 1}, \ldots, d p^{(K-1) S(L-1)}\right), d e^{K(S-1)}, d e^{K S}
\end{array}\right\} .
$$

Given any $\bar{\xi} \in \bar{\Xi}(\bar{E})$ and associated $\xi(\alpha)$, apply the following column operations (we identify columns in the obvious way) to the matrix $D_{\pi} \Phi(\pi ; \xi(\alpha))$ :

a. For each $k s$, add to column $d p^{k s L}$ the sum of the columns $\left\{d p^{k s 1}, \ldots, d p^{k s(L-1)}\right\}$ multiplied by the corresponding $\frac{\bar{p}^{s \ell}}{\bar{p}^{s L}}$,

b. Subtract column $d p^{k s k}$ from column $d p^{k^{\prime} s k}$ to eliminate all the (collinear) columns $d p^{k^{\prime}} s k, k^{\prime} \neq k$,

c. Use columns $d e^{K S}$ and $d e^{K(S-1)}$ to get rid of all the non-zero coefficients in the last two rows.

Fact 3. $\quad$ For each $\alpha \neq 0, D_{\pi^{d}} W(\bar{\pi} ; \xi(\alpha))$ has full rank. Moreover, modulo a rearrangement of the columns,

$$
\left.\begin{array}{rl}
D_{\pi} \widetilde{\Phi}(. ; \xi(\alpha))= & {\left[\begin{array}{cc}
D_{\pi \backslash d} \widetilde{\Theta}(. ; \xi(\alpha)) & D_{\pi^{d}} \widetilde{\Theta}(. ; \xi(\alpha)) \\
{[0]} & {\left[D_{\pi^{d}} W(. ; \xi(\alpha))\right]}
\end{array}\right]} \\
& {\left[\begin{array}{c}
D_{\pi^{\backslash d, L}} \widetilde{\zeta}^{\circ}(. ; \xi(\alpha)) \\
D_{\pi^{\backslash d, L}} \widetilde{G}_{n d}^{\backslash 0}(. ; \xi(\alpha))
\end{array}\right]\left[\begin{array}{c}
D_{\pi^{L}} \widetilde{\zeta}^{\circ}(. ; \xi(\alpha)) \\
D_{\pi^{L}} \widetilde{G}_{n d}^{\backslash 0}(. ; \xi(\alpha))
\end{array}\right]\left[\begin{array}{c}
D_{\pi^{d}} \zeta^{\circ}(. ; \xi(\alpha)) \\
D_{\pi^{d}} G_{n d}^{\backslash 0}(. ; \xi(\alpha))
\end{array}\right],} \\
{[0]} & {[0]}
\end{array}\right],
$$

Proof of Fact 3. Given $\alpha \neq 0, D_{\pi} W(. ; \xi(\alpha))$ (omitting the first $L$ columns, and the ones in the collection $\left\{L^{*}+1, \ldots, L^{*}+I\right\}$, which are identically zero) is given 
by

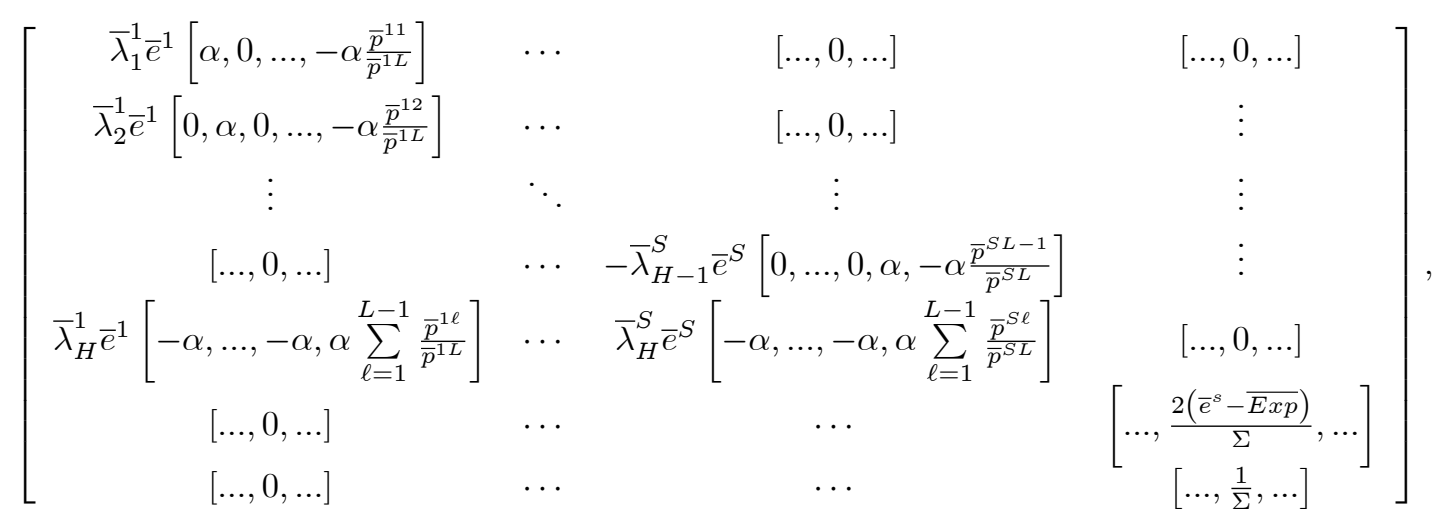

where we use the index $s$ to emphasize that all the variables are sunspot-invariant.

Each one of the first $\Sigma L$ columns (but the one referred to good $s L$ ) has just two non-zero coefficients: the one of agent $h$ such that $s \ell=f(h)$ and the one of agent $H$. By Fact 2, there is no loss of generality in assuming that $\left[\bar{\lambda}_{1}^{1}, \bar{\lambda}_{1}^{2}\right]$ and $\left[\bar{\lambda}_{H}^{1}, \bar{\lambda}_{H}^{1}\right]$ are not collinear. Hence, the first $H$ rows are linearly independent. The last two rows are clearly linearly independent of all the other rows. Hence, $\operatorname{rank} D_{\pi^{d}} W(. ; \xi(\alpha))=H+2$, for each $\alpha \neq 0$. Due to the column operations $(a-c)$, all the other columns of $D_{\pi} W(. ; \xi(\alpha))$ are identically zero. Hence, $D_{\pi} W(. ; \xi(\alpha))$ has the structure displayed above.

We now show that $D_{\pi \backslash d} \widetilde{\Theta}(. ; \xi(\alpha))$ has full rank $\left(L^{*}+I-1\right)$. We proceed in two steps. Fact 4 shows that, modulo a perturbation of $\bar{u}_{1}, D_{\pi \backslash d, L} \widetilde{\zeta}^{\circ}(. ; \xi(\alpha))$ has full rank. Then, Fact 5 will show that, generically, $D_{\pi \backslash d} \widetilde{\Theta}(. ; \xi(\alpha))$ has full rank, too.

Let $\zeta^{\circ}(. ; \xi(\alpha))$ be obtained deleting the market clearing eqs. for all the commodities included in the collection $\pi^{d}$ and commodity $L$ in states $0, K(S-1)$ and $K S$. Evidently, $\zeta^{\circ}(. ; \xi(\alpha))=0$ implies market clearing for all the assets and commodities.

Fact 4. For each $\alpha \neq 0$ and close enough to $0, D_{\pi \backslash d, L} \widetilde{\zeta}^{\circ}(. ; \xi(\alpha))$ has full rank, $\left(L^{*}+I-1-\Sigma\right)$.

Proof of Fact 4.. $\quad$ To simplify, we compute the rank of $D_{\pi \backslash d, L} \widetilde{\zeta}^{\circ}(. ; \xi(\alpha))$ at $\alpha=0$. Once we have shown that this rank is full, continuity of $\operatorname{det} D_{\pi \backslash d, L} \widetilde{\zeta}^{\circ}(. ; \xi(\alpha))$ in $\alpha$ will imply the stated property.

By Fact $1, D_{\pi} z_{1}=-\left[\begin{array}{cc}B_{1}^{1} & B_{1}^{2}\end{array}\right] D_{\pi} F O C_{1}$. Bear in mind that, at $\alpha=0$, the last $(\Sigma+1)$ rows of $D_{\pi} F O C_{1}$ are identically zero, so that we can drop them whenever convenient.

Given the structure of $D_{\pi} F O C_{1}$, displayed above (before the proof of Lemma $2)$, the column operations described above affect:

1. the rows referred to the commodities included in the collections $\pi^{d}$ and $\pi^{L}$,

2. the last $\Sigma$ columns of the rows referred to states $K(S-1), K S$.

Apply the column operations $(a, b, c)$ described above to $D_{\pi} F O C_{1}$. Drop the rows and columns referred to the commodities included in $\pi^{d}$ and to commodity $L$ in states $0, K(S-1)$ and $K S$. For each $k s \neq K(S-1), K S$, replace the column 
referred to commodity $L$ with the one referred to the corresponding exchange rates, obtaining the matrix

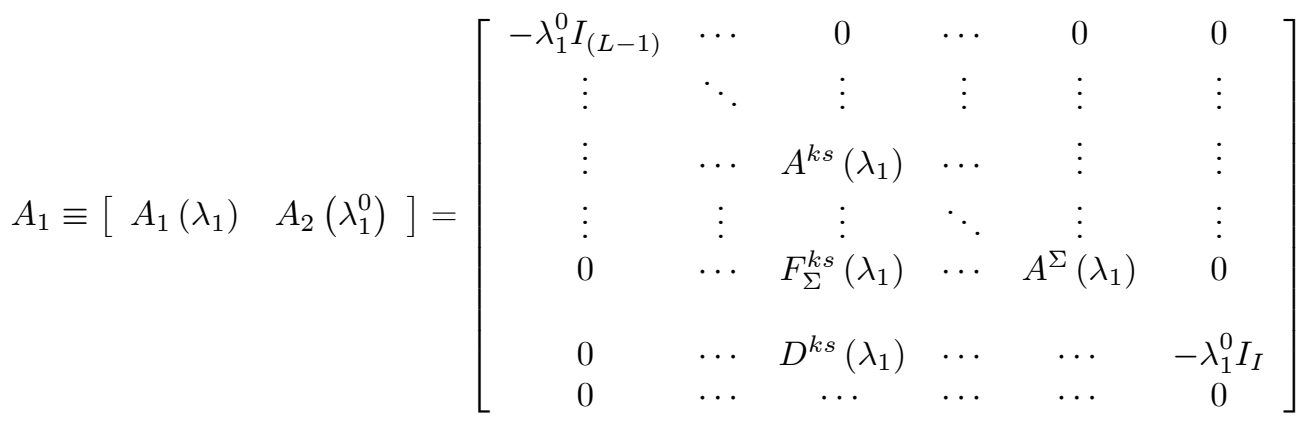

Evidently,

$$
D_{\pi \backslash d, L} \widetilde{z}_{1}^{\circ}(.)=-B_{1}^{1^{\circ}}\left[\begin{array}{ll}
A_{1}\left(\lambda_{1}\right) & A_{2}\left(\lambda_{1}^{0}\right)
\end{array}\right],
$$

where $B_{1}^{1^{\circ}}$ is obtained dropping the rows and columns of the commodities in the collection $\pi^{d}, 0 L, K(S-1) L$, and $K S L$.

The square $(L-1)$-dimensional matrix $A_{1}\left(\bar{\lambda}_{1}\right)$ is independent of $\omega_{1}$, while it is a linear function of $\lambda_{1}$. For $k s \neq K(S-1), K S$,

$$
A^{k s}\left(\lambda_{1}\right) \equiv\left[\begin{array}{cc}
-\lambda_{1}^{s} \bar{e}^{s} I_{(L-2)} & -\lambda_{1}^{s}\left[\bar{p}^{k s \backslash \ell(\sigma), L}\right]^{T} \\
0 & -\lambda_{1}^{s} \bar{p}^{k s L}
\end{array}\right],
$$

where $\left[\bar{p}^{k s \backslash \ell(\sigma), L}\right]$ is the $(L-2)$-dimensional vector obtained dropping $\bar{p}^{k s \ell(\sigma)}$ and $\bar{p}^{k s L}$. Hence, $A^{k s}\left(\lambda_{1}\right)$ has full rank $(L-1)$. For $k s=K(S-1), K S, A^{k s}\left(\lambda_{1}\right) \equiv$ $\left[-\lambda_{1}^{s} \bar{e}^{s} I_{(L-1)}\right] \cdot D^{k s}\left(\lambda_{1}\right)$ is a linear combination of the columns $-\lambda_{1}^{k s}\left[r_{A}^{s}\right]^{T}$. The other off-diagonal nonzero components of $A_{1}\left(\lambda_{1}\right)$ are the matrices $F_{\sigma}^{k s}\left(\lambda_{1}\right)$, which are non-trivial only for the rows associated with the commodities of states $K(S-1)$ and $K S$. The ones associated with $K S$ are $\left.F_{K S}^{k s}\left(\lambda_{1}\right)=\left[\begin{array}{ll}0 I_{(L-1)} & \lambda_{1}^{s}\left[\bar{p}^{\sigma \backslash \ell(\sigma)}\right.\end{array}\right]^{T}\right]$. The ones associated with $K(S-1)$ are

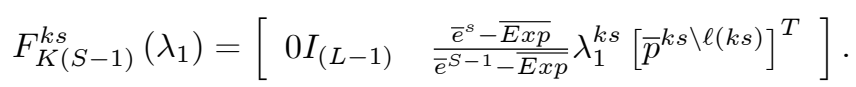

Each one of these matrices is a linear function of $\lambda_{1}$, independent of $\omega_{1}$. All the $(L-1)$-dimensional square matrices on the diagonal have full rank. Hence, given its block triangular structure structure, $\left[\begin{array}{ll}A_{1}\left(\lambda_{1}\right) & A_{2}\left(\lambda_{1}\right)\end{array}\right]$ has full rank $\left(L^{*}+I-\Sigma-1\right)$. As reported in Fact $1, B_{1}^{1^{\circ}}$ has full rank, so that the same holds for $\left[B_{1}^{1^{\circ}}\left[\begin{array}{ll}A_{1}\left(\lambda_{1}\right) & A_{2}\left(\lambda_{1}\right)\end{array}\right]\right]$. Since

$$
\begin{aligned}
& D_{\pi \backslash d, L} \widetilde{\zeta}^{\circ}(.)=-\left[B_{1}^{1^{\circ}}\left[\begin{array}{ll}
A_{1}\left(\lambda_{1}\right) & A_{2}\left(\lambda_{1}\right)
\end{array}\right]+\sum_{h>1} D_{\pi \backslash d, L} \widetilde{z}_{h}^{\circ}(.),\right. \\
& \operatorname{rank} D_{\pi \backslash d, L} \widetilde{\zeta}^{\circ}(.)=\operatorname{rank}\left[B_{1}^{1^{\circ}}\left[A_{1}\left(\frac{\lambda_{1}}{\lambda_{1}^{\circ}}\right) A_{2}(1)\right]\right]^{-1} D_{\pi \backslash d, L} \widetilde{\zeta}^{\circ}(.) \\
& =\operatorname{rank}\left[B_{1}^{1^{\circ}}\left[\begin{array}{ll}
A_{1}\left(\frac{\lambda_{1}}{\lambda_{1}^{0}}\right) & A_{2}(1)
\end{array}\right]\right]^{-1}\left[\sum_{h>1} D_{\pi \backslash d, L} \widetilde{z}_{h}^{\circ}(.)\right] \\
& -\lambda_{1}^{0}\left[I_{\left(L^{*}+I-\Sigma-1\right)}\right] \text {. }
\end{aligned}
$$


If $\operatorname{det} D_{\pi \backslash d, L} \widetilde{\zeta}^{\circ}()=0,. \lambda_{1}^{0}$ is an eigenvalue of the matrix just described. The perturbation of the utility function described above allows us to change arbitrarily $\lambda_{1}^{0}$, without affecting the vector $\frac{\lambda_{1}}{\lambda_{1}^{0}}$. Given that, for every square matrix, eigenvalues are locally unique, modulo an arbitrarily small perturbation of $u_{1}$, $\operatorname{det} D_{\pi \backslash d, L} \widetilde{\zeta}^{\circ}() \neq$.0 .

Fact 5. Modulo an arbitrarily small perturbation of $\xi(\alpha), D_{\left(\pi^{\backslash d, L}, \pi^{L}\right)} \widetilde{\Theta}($. has full rank.

Proof of Fact 5. We can exploit the same perturbation of $\left(u_{1}, \omega_{1}\right)$ used in the proof of Lemma 2 above to affect the matrix $D_{\pi^{L}} \widetilde{G}_{n d}^{\backslash 0}($.$) , without affecting the$ equilibrium and the matrices $D_{\pi \backslash d, L} \widetilde{z}_{1}^{\circ}($.$) and, hence, D_{\pi \backslash d, L} \widetilde{\zeta}^{\circ}($.$) .$

The columns operations $(a)$ described above turn the matrix $D_{\pi^{L}} G_{n d}^{\backslash 0}($.$) into$

$$
D_{\pi_{L}} \widetilde{G}_{n d}^{\backslash 0}(.)=\left[\begin{array}{ccc}
\ddots & \vdots & \vdots \\
\vdots & \bar{e}^{k s} \sum_{h} \widehat{\omega}_{h}^{s L} & \vdots \\
\vdots & \vdots & \ddots
\end{array}\right]+\left[\begin{array}{ccc}
\ddots & \vdots & \vdots \\
\vdots & \sum_{\ell=1}^{L-1} \bar{p}^{s \ell} & \vdots \\
\vdots & \vdots & \ddots
\end{array}\right]
$$

Given that $D_{\pi \backslash d, L} \widetilde{\zeta}^{\circ}($.$) has full rank, the following matrix is well-defined$

$$
\begin{aligned}
& {\left[\begin{array}{cc}
D_{\pi \backslash d, L} \widetilde{\zeta}^{\circ}(.) & D_{\pi^{L}} \widetilde{\zeta}^{\circ}(.) \\
D_{\pi \backslash d, L} \widetilde{G}_{n d}^{\backslash 0}(.) & D_{\pi^{L}} \widetilde{G}_{n d}^{\backslash 0}(.)+\delta I_{\Sigma}
\end{array}\right]\left[\begin{array}{cc}
I_{L^{*}+I-\Sigma-1} & -\left[D_{\pi \backslash d, L} \widetilde{\zeta}^{\circ}(.)\right]^{-1} D_{\pi^{L}} \widetilde{\zeta}^{\circ}(.) \\
0 & I_{\Sigma}
\end{array}\right]} \\
& =\left[\begin{array}{cc}
D_{\pi \backslash d, L} \widetilde{\zeta}^{\circ}(.) & 0 \\
D_{\pi \backslash d, L} \widetilde{G}_{n d}^{\backslash 0}(.) & -\left[D_{\pi \backslash d, L} \widetilde{G}_{n d}^{\backslash 0}(.)\left[D_{\pi \backslash d, L} \widetilde{\zeta}^{\circ}(.)\right]^{-1} D_{\pi^{L}} \widetilde{\zeta}^{\circ}(.)+D_{\pi^{L}} \widetilde{G}_{n d}^{\backslash 0}(.)\right]
\end{array}\right] .
\end{aligned}
$$

The (sunspot-invariant) perturbation used in the proof of Lemma 2 allows us to change $D_{\pi^{L}} \widetilde{G}_{n d}^{\backslash 0}($.$) by a term \delta I_{\Sigma}$, without affecting anything else. As already discussed there, this implies that we can find an arbitrarily small perturbation such that, at the associated $\delta$, the matrix above has full rank $\Sigma$. It follows that, for some economy $(\widetilde{u}, \widetilde{\omega})$, arbitrarily close to $(\bar{u}, \omega(\alpha)), D_{\pi} \widetilde{\Phi}($.$) has full rank.$

Proof of Theorem 4. By Fact 2, we can pick an economy $\bar{\xi}$ in the generic set such that, at a regular nonsunspot equilibrium $\bar{\pi}, \quad \frac{\bar{\lambda}_{1}^{1}}{\bar{\lambda}_{1}^{2}} \neq \frac{\bar{\lambda}_{H}^{1}}{\bar{\lambda}_{H}^{2}}$. Pick any open nbd $V(\bar{\xi})$. By Fact $3-5$, we can construct an economy $(\widetilde{u}, \widetilde{\omega})$, arbitrarily close to $(\bar{u}, \omega(\alpha))$, such that $D_{\pi} \widetilde{\Phi}($.$) has full rank at its nonsunspot equilibrium \bar{\pi}$.

Let $\widetilde{\delta}$ be the perturbation of agent 1's endowment and consumption defined in Fact 5 . Define the economy $\left(\widetilde{u}, \omega^{\circ}\right)$ where $\omega_{1}^{s L^{\circ}} \equiv\left(\bar{\omega}_{1}^{s L}-\frac{\widetilde{\delta}}{\bar{e}^{s}}\right)$ and $\omega_{1}^{s \ell^{\circ}} \equiv \bar{\omega}_{1}^{s \ell}$, for each $s \ell \neq s L$ and $s=0$. For each $h>1, \omega_{h}^{\circ} \equiv \bar{\omega}_{h} . x^{\circ}$ is also identical to $\bar{x}$, but for $x_{1}^{s L^{\circ}} \equiv\left(\bar{x}_{1}^{s L}-\frac{\widetilde{\delta}}{\bar{e}^{s}}\right)$. For $\widetilde{\delta}$ sufficiently small, $\left(\widetilde{u}, \omega^{\circ}\right) \in V(\bar{\xi})$ and $\bar{\pi}$ is one of its regular nonsunspot equilibria.

Let $\omega^{\mu} \equiv \mu \widetilde{\omega}+(1-\mu) \omega^{\circ}$ and consider the set of economies $\xi^{\mu} \equiv\left(\widetilde{u}, \omega^{\mu}\right)$. Evidently, $\bar{\pi}$ with associated $x^{\circ}$ is a ME for each $\mu \in[0,1]$. The vector $\left(\lambda_{1}, \ldots, \lambda_{H}\right)$ is also $\mu$-invariant. On the other hand, the vector $\left(y_{1}^{\mu}, \ldots, y_{H}^{\mu}\right)$ is a linear function of $\mu$. 
It follows that $\operatorname{det} D_{\pi} \Phi\left(\bar{\pi} ; \xi^{\mu}\right)$ is a polynomial in $\mu$. Given that $\operatorname{det} D_{\pi} \Phi\left(\bar{\pi} ; \xi^{1}\right) \neq 0$, for an open and dense subset (of full Lebesgue measure) of [0,1], $\operatorname{det} D_{\pi} \Phi\left(\bar{\pi} ; \xi^{\mu}\right) \neq$ 0 . Hence, we can pick $\mu$ such that $\xi^{\mu} \in V(\bar{\xi})$ and $\operatorname{det} D_{\pi} \Phi\left(\bar{\pi} ; \xi^{\mu}\right) \neq 0$. This establishes density. Given that $\operatorname{det} D_{\pi} \Phi($.$) is a continuous function, the openness part$ of the Thm. follows immediately.

Proof of Corollary 1. Consider a sunspot-invariant vector $\bar{e}$ and $\widehat{e}$ such that $\widehat{e}^{k s}=\bar{e}^{s}+\varepsilon^{k s}$ and, for each $s, \operatorname{Exp}\left(\varepsilon^{k s} \mid s\right)=0$. Then, $\widehat{e}$ is a mean-preserving spread of $\bar{e}$. To apply the argument of Thm. 4 to this set-up, we just need to replace, in the definition of $\Phi(\pi ; \xi, \bar{V}, \overline{v a r}, \overline{\operatorname{Exp}})$, the two eqs. $\operatorname{var}(e)=\overline{\operatorname{var}}, \operatorname{Exp}(e)=\overline{\operatorname{Exp}}$, with the $2 S$ eqs. $\operatorname{var}(e \mid s)=\overline{v a r}$ and $\operatorname{Exp}(e \mid s)=\overline{\operatorname{Exp}}$. It $\mathrm{s}$ easy to verify that the proof works with some, mainly notational, adjustments, once we restrict the analysis to economies with $2 \leq H \leq S(L-3)$ agents.

\section{PROOF OF THEOREM 5}

We consider economies without sunspots, so that $L^{*} \equiv(S+1) L$. As well known, in GEI models, the fundamental difficulty in the proof of the existence of equilibria is due to the possible drop of rank of the payoff matrix when some variables (here, exchange rates) hit some critical set of values. The canonical approach to deal with this problem is in Duffie and Shafer [11]. Here, we just need to modify slightly their argument to take care of the additional equilibrium conditions given by the monetary equations $G_{d}^{\backslash 0}()=$.0 .

We start reformulating in a more convenient form the individual optimization problems. Exploiting "Cass' trick", agent 1's optimization problem can be recasted as: Given $(p, \mu, e) \in \mathbf{P} \equiv\left\{\mathbb{R}_{++}^{L^{*}} \times \mathbb{R}_{++}^{S+1} \times \mathbb{R}_{++}^{S+1} \mid \mu^{0}=1, e^{0}=1\right\}$, a smooth manifold of dimension $\left(L^{*}+2 S\right)$, agent 1 solves optimization problem

$$
\text { choose } \quad x_{1} \in \arg \max u_{1}\left(x_{1}\right) \quad \text { subject to } \quad \sum_{s \geq 0} \mu^{s} e^{s} p^{s} x_{1}^{s}=1 \text {. }
$$

It is well known that, under the maintained assumptions, the solution to (2) exists, it is $C^{1}$ at each $(\bar{p}, \bar{\mu}, \bar{e})>>0$, and satisfies the boundary condition: for each $\left\{\left(p^{v}, \mu^{v}, e^{v}\right)\right\}_{v=1}^{\infty}$, with $\left(p^{v}, \mu^{v}, e^{v}\right) \in \mathbf{P}$ for each $v$ and such that $\left(p^{v}, \mu^{v}, e^{v}\right) \rightarrow$ $(\bar{p}, \bar{\mu}, \bar{e}) \notin \mathbf{P}$, the associated sequence $\left\{z_{1}\left(p^{v}, \mu^{v}, e^{v}\right)\right\}_{v=1}^{\infty}$ satisfies $\frac{1}{\left\|z_{1}\left(p^{v}, \mu^{v}, e^{v}\right)\right\|} \rightarrow$ 0 .

For $h>1$, given $(p, \mu, e) \in \mathbf{P}$ and a linear subspace $\gamma$ of dimension $I$ in $\mathbb{R}^{S}$, replace $h$ 's optimization problem with

$$
\begin{aligned}
\text { choose } x_{h} \in \arg \max u_{h}\left(x_{h}\right) \quad \text { subject to } & \sum_{s} \mu^{s} e^{s} p^{s} z_{h}^{s}=0 \\
& {\left[\begin{array}{c}
e^{1} p^{1} z_{h}^{1} \\
\vdots \\
e^{S} p^{S} z_{h}^{S}
\end{array}\right] \in \gamma . }
\end{aligned}
$$

As well known, under our assumptions, the optimal solution to (3) exists, is $C^{1}$ at each $(\bar{p}, \bar{\mu}, \bar{e}) \in \mathbf{P}$, and, if $\bar{\gamma}=\operatorname{span}[R(\bar{e})]$, it is also an optimal solution to (2). 
Following Duffie and Shafer [11], given $\left(\bar{M}_{A}, \bar{M}_{B}\right)$, a vector $(\bar{p}, \bar{\mu}, \bar{e}, \bar{\gamma})$ with associated allocation $\bar{x}$ is a pseudo-equilibrium if

i. $\quad \bar{x}_{1}$ is an optimal solution to (2), and $\bar{x}_{h}$ is an optimal solution to (3), for each $h>1$,

ii. $\quad \sum_{h}\left(\bar{x}_{h}-\omega_{h}\right)=0$,

iii. $\quad G_{d}^{s}\left(\bar{M}_{A}, \bar{M}_{B}, \bar{p}, \bar{e}\right)=0$, for each $s>0$,

iv. $\quad \operatorname{span}[R(\bar{e})] \subset \bar{\gamma}$.

In this definition, the monetary eqs. are satisfied only at $s>0$. However, modulo a normalization, pseudo-equilibria such that $\operatorname{span}[R(\bar{e})]=\bar{\gamma}$ are actual $\mathrm{ME}$, according to Definition 1 in the text, as established below.

Fact 6 . Given $\left(\bar{M}_{A}, \bar{M}_{B}\right)$, let $(\bar{p}, \bar{\mu}, \bar{e}, \bar{\gamma})$ with associated allocation $\bar{x}$ be a pseudo-equilibrium with $\operatorname{span}[R(\bar{e})]=\bar{\gamma}$. Then, $\widetilde{\pi}$ with

$i . \quad \widetilde{p}^{0}=\nu \bar{p}^{0}$ and $\left(\widetilde{p}^{s}, \widetilde{e}^{s}\right)=\left(\bar{p}^{s}, \bar{e}^{s}\right)$ for each $s>0$,

ii. $\quad \widetilde{q}_{A}=v[\bar{\mu}]^{T} R_{A}(\bar{e})$ and $\widetilde{q}_{B}=\frac{\bar{M}_{B}^{0}}{\bar{p}^{0} \sum_{h=H_{A}+1}^{H} \omega_{h}^{0}}[\bar{\mu}]^{T} R_{B}$,

iii. $\quad \widetilde{e}^{0}=\frac{M_{B}^{0}}{\nu \bar{p}^{0} \sum_{h=H_{A}+1}^{H} \omega_{h}^{0}}$,

for $v \equiv \frac{M_{A}^{0}}{\bar{p}^{0} \sum_{h=1}^{H_{A}} \omega_{h}}$, is a ME given $G_{d}()=$.0 , for some $\left(-\sum_{h>1}^{H} \bar{y}_{h}, \bar{y}_{2}, \ldots, \bar{y}_{H}\right)$.

Proof. Given that the asset prices $\widetilde{q}$ satisfy the noarbitrage conditions: $\widetilde{q}_{B}=$ $\frac{M_{B}^{0}[\bar{\mu}]^{T} R_{B}}{\bar{p}^{0} \sum_{h=H_{A}+1}^{H} \omega_{h}^{0}}$ and $\widetilde{e} 0 \widetilde{q}_{A}=\frac{M_{B}^{0}[\bar{\mu}]^{T} R_{A}(\widetilde{e})}{\bar{p}^{0} \sum_{h=H_{A}+1}^{H} \omega_{h}^{0}}$, it is straightforward to show that, for $h>1$, the budget constraints of (3) and (1) coincide in commodity allocation space, given the prices defined above. For $h=1$, at the given prices, the set of feasible consumption bundle of problem (1) is contained in the one of problem (2). By construction, $\left(\bar{x}_{1}, \bar{y}_{1}\right)$ is a feasible solution to problem (1) at $\widetilde{\pi}$, while $\bar{x}_{1}$ is the optimal solution to problem $(2)$ at $(\bar{p}, \bar{\mu}, \bar{e})$. Hence, $\left(\bar{x}_{1}, \bar{y}_{1}\right)$ must also be the optimal solution to problem (1). Therefore, $\widetilde{\pi}$ is a ME.

Given Fact 6, we just need to show that pseudo-equilibria with $\operatorname{rank} R(\bar{e})=I$ generically exist. Let $\Gamma^{S I}$ be the Grassmannian manifold of subspaces of $\mathbb{R}^{S}$ of dimension $I . \Gamma^{S I}$ is a $C^{\infty}$, compact manifold without boundary of dimension $(S-I) I$. Let $P_{\phi}$ be the permutation matrix induced by the permutation $\phi$ of the indexes $s=\{1, \ldots, S\}$ and $\Phi$ be their set. If $\gamma \in \Gamma^{S I}$, there is $\bar{\phi} \in \Phi$ and a $(S-I) \times I$-dimensional matrix $A$ such that $\gamma=\left\{w \in \mathbb{R}^{S} \mid[I \mid A] P_{\bar{\phi}} w=0\right\}$. Moreover, given $\bar{\phi}$, there is a unique matrix $A$ with the stated properties. Finally, let

$W_{\phi}=\left\{\gamma \in \Gamma^{S I} \mid \exists\right.$ a $(S-I) \times I$ matrix $A$ such that $\left.\gamma=\left\{w \in \mathbb{R}^{S} \mid[I \mid A] P_{\phi} w=0\right\}\right\}$,

and $\varphi_{\phi}, \varphi_{\phi}: W_{\phi} \rightarrow \mathbb{R}^{(S-I) I}$, be the map associating with each element in $W_{\phi}$ the matrix $A$ such that $\gamma=\left\{w \in \mathbb{R}^{S} \mid[I \mid A] P_{\bar{\phi}} w=0\right\}$. Then, $\left\{W_{\phi}\right\}_{\phi}$ defines an open cover of $\Gamma^{S I}, \varphi_{\phi}$ is a homeomorphism of $W_{\phi}$ onto $\mathbb{R}^{(S-I) I}$, and $\left\{W_{\phi}, \varphi_{\phi}\right\}_{\phi}$ is an atlas for $\Gamma^{S I}$ (these are Facts 1-3 in [11]). Define as $Z(p, \mu, e, \gamma)$ the system of aggregate excess demand functions, $Z(p, \mu, e, \gamma) \equiv z_{1}(p, \mu, e, 1)+$ $\sum_{h>1} z_{h}\left(p, \mu, e, \omega_{h}, \gamma\right)$. Evidently, $Z($.$) is C^{1}$ at $(\bar{p}, \bar{\mu}, \bar{e}, \bar{\gamma})$ with $(\bar{p}, \bar{\mu}, \bar{e})>>0$, and satisfies the analogous of the boundary conditions established above for agent 1. Moreover, $Z(p, \mu, e, \gamma)=0$ only if $\sum_{s} \mu^{s} e^{s} p^{s} \omega_{1}^{s}=1$. Also, define 
$K_{\phi}(\mu, e, r, \gamma)=\left[I \mid \varphi_{\phi}(\gamma)\right] P_{\phi} R(e)$, with $K_{\phi}: \mathbb{R}_{++}^{S} \times \mathbb{R}_{++}^{S} \times \mathbb{R}^{S I} \times W_{\phi} \rightarrow \mathbb{R}^{(S-I) I}$. By Fact 7 in [11], $K_{\phi}$ is a smooth map and $\operatorname{rank} D_{r} K_{\phi}=(S-I) I$. Define the pseudo-equilibrium map

$$
F\left(p, \mu, e, r, \gamma, \omega, M_{A}^{\backslash 0}, M_{B}^{\backslash 0}\right)=\left[\begin{array}{c}
Z(p, \mu, \gamma, \omega) \\
K_{\phi}(\mu, e, r, \gamma) \\
G_{d}^{\backslash 0}\left(p, e, \omega, M_{A}^{\backslash 0}, M_{B}^{\backslash 0}\right)
\end{array}\right],
$$

and the set

$\Lambda \equiv\left\{\left(p, \mu, e, r, \gamma, \omega, M_{A}^{\backslash 0}, M_{B}^{\backslash 0}\right) \mid F()=\right.$.0 , for $\phi$ such that $\left.\gamma=\left\{y \in \mathbb{R}^{S} \mid[I \mid A] P_{\phi} y=0\right\}\right\}$.

Fact 7. Under the maintained assumptions, for each $\phi$,

a. $\quad 0$ is a regular value of $F$,

b. $\quad \Lambda$ is a submanifold of $\mathbb{R}_{++}^{L^{*}} \times \mathbb{R}_{++}^{S} \times \mathbb{R}_{++}^{S} \times \mathbb{R}^{S I} \times W_{\phi} \times \mathbb{R}_{++}^{H L^{*}} \times \mathbb{R}_{++}^{2 S}$

without boundary of dimension $H L^{*}+S I+2 S$,

c. the projection maps $\operatorname{Pr}: \Lambda \rightarrow \mathbb{R}_{++}^{H L^{*}} \times \mathbb{R}_{++}^{2 S} \times \mathbb{R}^{S I}$ is proper.

Proof of Fact \%. All the properties can be established as in Fact 10 in [11], once we observe that the matrix

$$
D_{\left(\omega_{1}, r, M_{A}^{\backslash 0}, M_{B}^{\backslash 0}\right)} F(.)=\left[\begin{array}{cccc}
D_{\omega_{1}} Z & 0 & 0 & 0 \\
0 & D_{r} K_{\phi} & 0 & 0 \\
-\Psi^{\backslash 0}(p) & 0 & -\operatorname{diag}(e) & -I \\
-\Psi^{\backslash 0}(p) & 0 & -I & 0
\end{array}\right]
$$

has full rank. Given that $\operatorname{rank} D_{r} K_{\phi}=(S-I) I$, the top left matrix has full rank. The bottom right submatrix has full rank, too. Hence, $D_{\left(\omega_{1}, r, M_{A}^{\backslash 0}, M_{B}^{\backslash 0}\right)} F($.$) has$ full rank.

Proof of Theorem 5. By Fact 7, Pr : $\Lambda \rightarrow \mathbb{R}_{++}^{H L^{*}} \times \mathbb{R}_{++}^{2 S} \times \mathbb{R}^{S I}$ is a proper map between two manifolds, without boundaries, of the same dimension. $\mathbb{R}_{++}^{H L^{*}+2 S} \times \mathbb{R}^{S I}$ is connected. Hence, applying mod 2 degree theory, if there is a regular value $\xi \in \Xi$ of $\operatorname{Pr}($.$) such that the pseudo-equilibrium is unique, then$ there is a pseudo-equilibrium for each $\xi \in \Xi$. Pick any $\bar{\omega} \in \mathbb{R}_{++}^{H L^{*}}$ such that $\bar{\omega}$ is a Pareto optimal allocation, any $\bar{e} \neq[\mathbf{1}]$, any collection $r=\left(r^{1}, \ldots, r^{I}\right)$ such that $r^{1}=r^{I_{A}+1}=[\mathbf{1}]$, rank $R(\bar{e})=I$, and such that the last $I$ rows of $R(\bar{e})$ have full rank (all of this can be done under $A 2$ ). We will choose $\left(\bar{M}_{A}, \bar{M}_{B}\right)$ appropriately later on.

Pareto optimality obviously implies that, for each $\left(\bar{M}_{A}^{\backslash 0}, \bar{M}_{B}^{\backslash 0}\right)$, there is a unique equilibrium allocation. Agent 1's first order conditions fix the relative prices $\frac{\bar{p}^{s}}{\bar{p}^{s 1}}$. Fix $\bar{\mu}=[\mathbf{1}]$ and normalize prices so that $\sum_{s} \bar{\mu}^{s} \bar{e}^{s} \bar{p}^{s} \bar{\omega}_{1}^{s}=1$. Finally, choose $\left(\bar{M}_{A}, \bar{M}_{B}\right)$ so that the monetary eqs. are satisfied at each $s>0$. Given that $\operatorname{rank} R(\bar{e})=I$, there is a unique solution to $[I \mid A] R(\bar{e})=0$. Then, it is immediate to verify that, given $\left(\bar{\omega},\left(\bar{M}_{A}^{\backslash 0}, \bar{M}_{B}^{\backslash 0}\right), r\right)$, there is a unique ME $(\bar{p}, \bar{\mu}, \bar{e}, \bar{\gamma})$.

We still need to show that this pseudo-equilibrium is regular. Given $\phi^{*}=i d$, let $G_{d}^{a \backslash 0}(p, e)$ and $G_{d}^{b \backslash 0}(p)$ be given by the first and last $S$ rows of $G_{d}^{\backslash 0}(p, e)$ 
and consider

$$
D_{(p, A, \mu, e)} F(.)=\left[\begin{array}{cccc}
D_{p} Z & 0 & D_{e} Z & D_{\mu} Z \\
0 & D_{A} K_{i d} & D_{e} K_{i d} & 0 \\
\Psi^{\backslash 0}\left(\sum_{h=1}^{H} \omega_{h}, \bar{e}\right) & 0 & D_{e} G_{d}^{a \backslash 0} & 0 \\
\Psi^{\backslash 0}\left(\sum_{h=H_{A}+1}^{H} \omega_{h}, \bar{e}\right) & 0 & 0 & 0
\end{array}\right],
$$

where $\Psi^{\backslash 0}\left(\sum_{h=1}^{H} \omega_{h}, \bar{e}\right)$ has the same structure as $\Psi^{\backslash 0}(p, \bar{e})$, but for $p^{s \ell}$ replaced by $\sum_{h=1}^{H} \omega_{h}^{s \ell}$. Similarly for $\Psi^{\backslash 0}\left(\sum_{h=H_{A}+1}^{H} \omega_{h}, \bar{e}\right)$.

Notice that $D_{A} Z=0$ because, given that, for each $h, \bar{z}_{h}$ is also the optimal solution to problem (2), $\bar{z}_{h}$ is the optimal solution to (3) for each $\gamma$. By the same argument, $(\mu, e)$ is relevant just because it enters the aggregate budget constraints $\sum_{s \geq 0} \mu^{s} e^{s} p^{s} x_{1}^{s}=1$ and $\sum_{s>0} \mu^{s} e^{s} p^{s} z_{h}^{s}=0$. Given that just the product $\left(\ldots, \mu^{s} e^{s}, \ldots\right)$ matters, it must be $D_{\mu} Z=D_{e} Z$. Finally, $D_{A} K_{i d}$ has rank $(S-I) I$, as shown in [11], too.

Assume that $D_{(p, A, \mu, e)} F($.$) is singular. Then, there is a nonzero vector$ $\left(v_{p}, v_{A}, v_{a}, v_{b}\right)$ such that $v^{T} D_{(p, A, \mu, e)} F()=$.0 . Given that $D_{A} K_{i d}$ has full row rank, it must be $v_{A}=0$. Given that $D_{\mu} Z=D_{e} Z, v_{p} D_{\mu} Z=0$ implies $v_{p} D_{e} Z=$ 0 . Given that $D_{e} G_{d}^{a \backslash 0}$ is a diagonal matrix of full rank (and $v_{p} D_{e} Z+v_{A} D_{e} K_{i d}=$ $0), v_{a}=0$. Hence, $D_{(p, \mu, e, A)} F($.) has full rank if and only if the submatrix

$$
N \equiv\left[\begin{array}{cc}
D_{p} Z & D_{\mu} Z \\
\Psi^{\backslash 0}\left(\sum_{h=H_{A}+1}^{H} \omega_{h}, \bar{e}\right) & 0
\end{array}\right]
$$

does. By a standard result, if $\left[B_{1}^{1} B_{1}^{2}\right]$ defines the first $L^{*}$ rows of $D_{\left(x_{1}, \lambda_{1}\right)} F O C_{1}$,

$D_{(p, \mu)} z_{1}=-\left[\begin{array}{ll}B_{1}^{1} B_{1}^{2}\end{array}\right]\left[\begin{array}{cccccc}-\bar{\lambda}_{1} I_{L} & \cdots & 0 & 0 & \cdots & 0 \\ \vdots & \vdots & \vdots & -\bar{\lambda}_{1} \bar{e}^{1}\left[\bar{p}^{1}\right]^{T} & \cdots & 0 \\ \vdots & \ddots & \vdots & \vdots & \ddots & \vdots \\ 0 & \cdots & -\bar{\lambda}_{1} \bar{\mu}^{S} \bar{e}^{S} I_{L} & 0 & \cdots & -\bar{\lambda}_{1} \bar{e}^{S}\left[\bar{p}^{S}\right]^{T} \\ -\bar{\mu}^{0} \bar{x}_{1}^{01} & \cdots & -\bar{\mu}^{S} \bar{e}^{S} \bar{x}_{1}^{S L} & -\bar{e}^{1} \bar{p}^{1} \bar{x}_{1}^{1} & \cdots & -\bar{e}^{S} \bar{p}^{S} \bar{x}_{1}^{S}\end{array}\right]$,

where $I_{L}$ is the $L$-dimensional identity matrix. For each $h>1$, we can rewrite (3) as

$\max u_{h}\left(x_{h}\right) \quad$ subject to $\sum_{s} \mu^{s} e^{s} p^{s} z_{h}^{s}=0, \quad\left[I_{S-I} \mid \varphi_{\phi}(\bar{\gamma})\right]\left[\ldots, e^{s} p^{s} z_{h}^{s}, \ldots\right]^{T}=0$,

so that, given that $\bar{z}_{h}=0$, for each $h>1$,

$D_{p} \sum_{h>1} z_{h}=-\sum_{h>1}\left[B_{h}^{1} B_{h}^{2}\right]\left[\begin{array}{c}-\operatorname{diag}\left(\ldots, I_{L} \times \nabla_{x_{h}^{s}} u_{h}\left(x_{h}\right)\right) \operatorname{diag}\left(\ldots, I_{L} \times\left[\frac{1}{p^{s}}\right]^{T}, \ldots\right) \\ -\left[I_{S-I} \mid \varphi_{\phi}(\bar{\gamma})\right][\ldots, 0, \ldots]^{T}\end{array}\right]$,

Similarly,

$$
D_{\mu} \sum_{h>1} z_{h}=-\sum_{h>1}\left[B_{h}^{1} B_{h}^{2}\right]\left[\begin{array}{ccc}
-\bar{\lambda}_{h}^{1} \bar{e}^{1}\left[\bar{p}^{1}\right]^{T} & \vdots & \vdots \\
\vdots & \ddots & \vdots \\
\vdots & \vdots & -\bar{\lambda}_{h}^{S} \bar{e}^{S}\left[\bar{p}^{S}\right]^{T} \\
0 & \cdots & 0
\end{array}\right] .
$$


Pareto efficiency of $\bar{x}$ implies that the gradients of the utility functions are collinear. It follows that

$$
\begin{aligned}
& {\left[\begin{array}{lll}
D_{p} Z & \left.D_{\mu} Z\right]
\end{array}\right]\left[\begin{array}{cccc}
{[0]^{T}} & 0 & \cdots & 0 \\
0 & {\left[\frac{\bar{p}^{11}}{\bar{\mu}^{1}}\right]^{T}} & \cdots & 0 \\
\vdots & \vdots & \ddots & \vdots \\
0 & \cdots & \cdots & {\left[\frac{\bar{p}^{S L}}{\bar{\mu}^{S}}\right]^{T}}
\end{array}\right]} \\
& \equiv\left[D_{p} Z D_{\mu} Z\right]\left[\begin{array}{c}
C \\
-I_{S}
\end{array}\right]=[0] .
\end{aligned}
$$

Then,

$$
\begin{aligned}
\operatorname{rankN} & =\operatorname{rankN}\left[\begin{array}{cc}
I_{L^{*}} & C \\
0 & -I_{S}
\end{array}\right] \\
& D_{p} Z \\
& =\operatorname{rank}\left[\Psi^{\backslash 0}\left(\sum_{h=H_{A}+1}^{H} \omega_{h}, \bar{e}\right)\left[\begin{array}{ccc}
\ddots & 0 & \ldots \\
\vdots & \frac{-\bar{e}^{s} \bar{p}^{s} \sum_{h=H_{A}+1}^{H} \omega_{h}^{s}}{\bar{\mu}^{s}} & \vdots \\
0 & \cdots & \ddots
\end{array}\right]\right] \\
& =L^{*}+S,
\end{aligned}
$$

because (see Thm. 1 in [11]) $D_{p} Z$ has full rank, and the bottom right submatrix has rank $S$. Hence, $\bar{\xi}$ is a regular value of $\operatorname{Pr}($.$) , so that, for each \xi \in \Xi$, there is a pseudo-equilibrium.

A standard argument (see Thm. 2 in [11]) shows that, therefore, there is an open, dense subset $\Xi_{d} \subset \Xi$, such that, for each $\xi \in \Xi_{d}$, there is a finite collection of pseudo-equilibria such that $\operatorname{rank} R(\bar{e})=I$, locally described by smooth functions. In view of Fact 6 , modulo a normalization, these are actually ME.

\section{REFERENCES}

1. Alonso, I.: Persistent, nonfundamental exchange rate fluctuations, Review of Economic Dynamics 7, 687-706 (2004).

2. Bacchetta, P., and E. Van Wincoop, Does exchange rate stability increase trade and welfare?, American Economic Review 90, 1093-1109 (2000).

3. Balasko, Y., and D. Cass, The structure of financial equilibrium with exogenous yields: The case of incomplete markets, Econometrica 57, 135163 (1989).

4. Bergin, P.R., H.-C.Shin, F. Templeton, and I. Tchakarov, Does exchange rate variability matter for welfare? A quantitative investigation of stabilization policies, European Economic Review 51, 1041-1058 (2007). 
5. Cass, D.: Sunspots and incomplete financial markets: The general case, Economic Theory 2, 341-358 (1992).

6. Cass, D., A. Citanna: Pareto improving financial innovation in incomplete markets, Economic Theory 11, 467-494 (1998).

7. Cheung Y.W., M. Chinn, A. G. Pascual: Empirical exchange rate models of the Nineties: Are any fit to survive?, Journal of International Money and Finance 24, 1150-1175 (2005).

8. Citanna, A., A. Kajii, A. Villanacci: Constrained suboptimality in incomplete markets: A general approach and two applications, Economic Theory 11, 495-521 (1998).

9. Citanna, A., K., Schmedders: Excess price volatility and financial innovation, Economic Theory 26, 559-588 (2005).

10. Devereux, M.B., C. Engels: Exchange rate pass-through, exchange rate volatility, and exchange rate disconnect, Journal of Monetary Economics 49, 913-940 (2002).

11. Duffie, D., W. Shafer: Equilibrium in incomplete markets: I. A basic model of generic existence, Journal of Mathematical Economics 14, 285300 (1985).

12. Elul, R.: Welfare effects of financial innovation in incomplete markets economies with several consumption goods, Journal of Economic Theory 65, 43-78 (1995).

13. Elul, R.: Welfare-improving financial innovation with a single good, Economic Theory 13, 25-40 (1999).

14. Geanakoplos, J., A. Mas-Colell: Real indeterminacy with financial assets, Journal of Economic Theory 47, 22-38 (1989).

15. Kareken J., N. Wallace: On the indeterminacy of equilibrium exchange rates, Quarterly Journal of Economics 96, 207-22 (1981).

16. King, R. G., N. Wallace, W. E. Weber: Nonfundamental uncertainty and exchange rates, Journal of International Economics 32, 83-108 (1992).

17. Magill, M., M. Quinzii: Real effects of money in general equilibrium, Journal of Mathematical Economics 21, 301-342 (1992).

18. Manuelli, R.E., J. Peck: Exchange rate volatility in an equilibrium asset pricing model, International Economic Review 31, 559-574 (1990).

19. Martin, A.: Endogenous multiple currencies, Journal of Money, Credit and Banking 38, 1 245-262 (2006).

20. Mas-Colell, A.: Three observations on sunspots and asset redundancy, in P. Dasgupta, D. Gale, O.D. Hart and E. Maskin (eds.), Economic Analysis of Markets and Games. Essays in honor of Frank Hahn, Cambridge: M.I.T. Press 1992. 
21. Meese, R. A., K. Rogoff: Empirical exchange rate models of the Seventies: Do they fit out of sample?, Journal of International Economics 14, 3-24 (1983).

22. Neumeyer, P.A.: Currencies and the allocation of risk: The welfare effects of a monetary union, American Economic Review 88, 246-258 (1998).

23. Pietra, T.: Indeterminacy in general equilibrium economies with incomplete financial markets. Mixed assets returns, Journal of Mathematical Economics 21, 155-172 (1992).

24. Pietra, T.: The structure of the set of sunspot equilibria in economies with incomplete financial markets, Economic Theory 2, 321-340 (1992).

25. Pietra, T.: The set of sunspot equilibria in economies with incomplete financial markets: variable asset prices, Economic Theory 18, 649-659 (2001).

26. Pietra, T.: Real indeterminacy, sunspots and Pareto inefficiency in economies with incomplete asset markets, Economic Theory 24, 687-699 (2004).

27. Polemarchakis, H.P.: Portfolio choice, exchange rates and indeterminacy, Journal of Economic Theory 46, 414-421 (1988).

28. Russel, S.: Quasi-fundamental exhange rate variation, Economic Theory 22, 111-140 (2003).

29. Salto, M., T. Pietra, Welfare and excess volatility of exchange rates, Università di Bologna, DSE Working Papers, June 2011.

30. Suda, J.-M. Tallon, A. Villanacci: Real indeterminacy of equilibria in a sunspot economy with inside money, Economic Theory 2, 309-319 (1992).

31. Werner, J.: Equilibrium in economies with incomplete financial markets, Journal of Economic Theory 36, 110-119 (1985). 


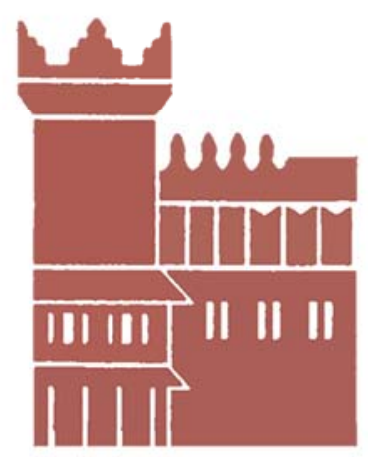

Alma Mater Studiorum - Università di Bologna DEPARTMENT OF ECONOMICS

Strada Maggiore 45

40125 Bologna - Italy

Tel. +39051 2092604

Fax +390512092664

http://www.dse.unibo.it 